\title{
Sensitivity Analysis for Rare Events based on Rényi Divergence
}

\author{
Paul Dupuis* \\ Division of Applied Mathematics, Brown University, Providence, RI 02912, USA
}

Markos A. Katsoulakis ${ }^{\dagger}$

Department of Mathematics and Statistics, University of Massachusetts Amherst, Amherst, MA 01002, USA

Yannis Pantazis ${ }^{\ddagger}$

Institute of Applied and Computational Mathematics, Foundation for Research and Technology - Hellas, Heraklion, 70013, Greece

Luc Rey-Bellet ${ }^{\S}$

Department of Mathematics and Statistics, University of Massachusetts Amherst, Amherst, MA 01002, USA

February 6, 2019

\begin{abstract}
Rare events play a key role in many applications and numerous algorithms have been proposed for estimating the probability of a rare event. However, relatively little is known on how to quantify the sensitivity of the probability with respect to model parameters. In this paper, instead of the direct statistical estimation of rare event sensitivities, we develop novel and general uncertainty quantification and sensitivity bounds which are not tied to specific rare event simulation methods and which apply to families of rare events. Our method is based on a recently derived variational representation for the family of Rényi divergences in terms of risk sensitive functionals associated with the rare events under consideration. Based on the derived bounds, we propose new sensitivity indices for rare events and relate them to the moment generating function of the score function. The bounds scale in such a way that we additionally develop sensitivity indices for large deviation rate functions.
\end{abstract}

\section{Introduction and Main Result}

Rare events play an important role in a wide range of applications. For example in insurance, finance and risk management, rare events play an outsized role due to potentially catastrophic consequences [1]. In queueing theory the probability of a buffer overflow often needs to be estimated [2, and in molecular dynamics, metastability effects play a crucial role in determining the behavior of the system [3. Similarly, extreme value theory studies events and statistical samples which are far from the typical observed [4. There is a large body of literature on rare event simulation and many different techniques have been developed to approximate the probability of a rare event. For example, importance sampling [5, 6, 7] transforms the distribution of random variables in order to make the rare event typical and corrects for bias using the likelihood ratio; interacting particle systems methods [8, 9] use many (dependent) copies of the system to speed the exploration of state space; splitting techniques [10, 11, 12, 13, decompose the problem of a single rare event into a sequence of not so rare events; and so on... Closely related are multi-level methods inspired primarily by statistical mechanics considerations, e.g. [14, as well as by rare events involving barrier crossing in molecular simulation, e.g. [15, 16].

In this paper we are primarily interested in the problem of uncertainty quantification (UQ) and in particular sensitivity analysis for rare events, a problem of practical importance whenever there is uncertainty in the parameters of the model or even in the model itself. This problem has rarely been addressed in the literature despite the fact that the statistics of rare events are often heavily influenced by the particular values of model parameters. The case of Poisson processes in the context of importance sampling for risk models was considered in [17, 18, while more recent work, 19, proposed

\footnotetext{
*dupuis@dam.brown.edu, Research supported in part by the Defense Advanced Research Projects Agency (DARPA) EQUiPS program (W911NF-15-2-0122), the Air Force Office of Scientific Research (AFOSR) (FA-9550-18-1-0214) and the National Science Foundation (DMS1317199)

${ }^{\dagger}$ markos@math.umass.edu, Research supported in part by the Defense Advanced Research Projects Agency (DARPA) EQUiPS program (W911NF-15-2-0122), the Air Force Office of Scientific Research (AFOSR) (FA-9550-18-1-0214) and the National Science Foundation (DMS$1515712)$

${ }^{\ddagger}$ pantazis@iacm.forth.gr

\$luc@math.umass.edu, Research supported in part by the National Science Foundation (DMS-1515712) and the Air Force Office of Scientific Research (AFOSR) (FA-9550-18-1-0214)
} 
importance sampling combined with splitting techniques in the context of Gaussian models and Malliavin calculus. Some examples using the likelihood ratio method are also discussed in [20].

Our approach in this paper is not based on any specific algorithm for rare event simulation but rather on novel information theoretic bounds. These bounds allow us to define a new sensitivity index that is independent of the particular event. Instead, the bounds hold for all rare events with probability above any fixed threshold. Specifically we utilize the Rényi family of relative entropies (a.k.a. Rényi divergence)as a measure of uncertainty between probability distributions and a new variational representation for risk-sensitive functionals in terms of Rényi relative entropy, derived by Atar et al. 21], to obtain general bounds on the uncertainty quantification and sensitivity analysis of families of rare events.

We first introduce the main objects of interest, namely sensitivity indices for probabilities of rare events, and discuss the challenges involved in their estimation. We then present the main result of the paper: bounds on the sensitivity indices for the families of events whose probability is at least $e^{-M}$, where $M$ is any fixed rare event threshold. At this stage we assume the degree of rarity is characterized by $M$ but later on a large deviation parameter will be introduced in Section 7.

Gradient sensitivity indices for rare events. Let $P^{\theta}$ be a family of probability measures parameterized by a vector $\theta \in \mathbb{R}^{K}$. We assume that $P^{\theta} \ll R$ where $R$ is a reference measure and we denote by $p^{\theta}=\frac{d P^{\theta}}{d R}$ the corresponding family of densities. We also assume that the mapping $\theta \mapsto p^{\theta}(x)$ satisfies suitable differentiability and integrability conditions in order to interchange integration and differentiation. For a rare event $A$ with $0<P^{\theta}(A) \ll 1$ we define the sensitivity index in the direction $v \in \mathbb{R}^{K}$ by

$$
S_{v}^{\theta}(A)=v^{T} \nabla_{\theta} \log P^{\theta}(A)=\frac{v^{T} \nabla_{\theta} P^{\theta}(A)}{P^{\theta}(A)},
$$

which describes the relative change of the quantity of interest $P^{\theta}(A)$ with respect to the parameter $\theta$ in the $v$ direction.

One of the simplest ways to estimate the sensitivity index (11) is by considering finite difference approximations for each partial derivative, i.e., considering all coordinate unit directions $v=e_{i} \in \mathbb{R}^{K}, i=1,2, \ldots, K$ :

$$
S_{e_{i}}^{\theta}(A) \approx \frac{\log P^{\theta+\epsilon e_{i}}(A)-\log P^{\theta}(A)}{\epsilon} .
$$

However, the cost of implementing such an approximation can be prohibitive, given the cost of estimating the small probabilities $P^{\theta+\epsilon e_{i}}(A), i=1,2, \ldots, K$. A variant of the likelihood ratio method can at least partially address this issue, as we discuss next.

Likelihood ratio method for rare events. The gradient sensitivity for expected values of observables can be computed using various methods, such as the likelihood ratio method and infinitesimal perturbation analysis, see e.g. [5]. While these methods are in principle applicable to the problem of computing the rare event sensitivity index (11) (as we show next in the context of likelihood ratio), they still require the use of some form of accelerated Monte Carlo simulation, for example importance sampling [5, to (possibly) obtain acceptable performance when rare events are involved.

We define the score function for the parametric family $P^{\theta}$ by

$$
W^{\theta}(x):=\nabla_{\theta} \log p^{\theta}(x),
$$

with the convention that $W^{\theta}(x)=0$ if $p^{\theta}(x)=0$. We also denote by $P_{\mid A}^{\theta}$ the probability $P^{\theta}$ conditioned on the event $A$, i.e., $P_{\mid A}^{\theta}(d x)=\frac{1}{P^{\theta}(A)} \chi_{A}(x) p^{\theta}(x) R(d x)$, where $\chi_{A}$ is the indicator function.

Under suitable conditions to ensure the interchangeability between integrals and derivatives, the sensitivity index for the rare event $A$ given in (11) can be rewritten as

$$
S_{v}^{\theta}(A)=\frac{v^{T} \nabla_{\theta} \int_{A} p^{\theta} d R}{P^{\theta}(A)}=\frac{v^{T} \int_{A} W^{\theta} d P^{\theta}}{P^{\theta}(A)}=\mathbb{E}_{P_{\mid A}^{\theta}}\left[v^{T} W^{\theta}\right]
$$

An algorithm that estimates (4) by combining the likelihood ratio method with importance sampling through interacting particles was recently developed in [20]. Both approaches, (2) and (4), are feasible only when an accelerated Monte Carlo scheme appropriate to the particular event $A$ has been designed. Therefore, sensitivity analysis methods for rare events which would apply to a whole class of events $A$ (or more generally expected values which are sensitive to rare events), would be a more practical computational tool. One approach to this end is to derive upper and lower bounds for $S_{v}^{\theta}(A)$ that can serve as new sensitivity indices. These are of course less accurate, but may be much easier to compute, and can be used to identify those parameters for which greater accuracy is not needed. We show next that the well-known Cramer-Rao type bounds are not useful for the sensitivity analysis of rare events.

Failure of Cramer-Rao type bounds. The sensitivity index for a regular, i.e., non-rare, observable has the form $\nabla_{\theta} \mathbb{E}_{P^{\theta}}[f]=\mathbb{E}_{P^{\theta}}\left[f W^{\theta}\right]$. This can be easily bounded using the Cramer-Rao inequality, 22] i.e.,

$$
\left|v^{T} \nabla_{\theta} \mathbb{E}_{P^{\theta}}[f]\right| \leq \sqrt{\operatorname{Var}_{P^{\theta}}[f]} \sqrt{v^{T} \mathcal{F}\left(P^{\theta}\right) v}
$$


where $\mathcal{F}\left(P^{\theta}\right)=\mathbb{E}_{P^{\theta}}\left[W^{\theta}\left(W^{\theta}\right)^{T}\right]$ is the Fisher information matrix. Applying the Cramer-Rao bound to a rare event $A$ yields

$$
\left|S_{v}^{\theta}(A)\right|=\frac{1}{P^{\theta}(A)}\left|\mathbb{E}_{P^{\theta}}\left[\chi_{A} v^{T} W^{\theta}\right]\right| \leq \sqrt{\frac{1-P^{\theta}(A)}{P^{\theta}(A)}} \sqrt{v^{T} \mathcal{F}\left(P^{\theta}\right) v} .
$$

Unfortunately, this (naive) sensitivity bound is rather useless since it scales as $P^{\theta}(A)^{-1 / 2}$. This can be very large for a rare event $A$, while one expects the sensitivity index to be of order $O(1)$.

Information-based sensitivity indices for rare events. In view of the difficulty of directly approximating (1D), the main contribution in this paper is to develop information-based bounds for the sensitivity indices defined in (11) that apply to families of rare events. The bounds involve only a single risk-sensitive functional for the score function $W^{\theta}$. While this quantity must be approximated, the bound does not require a different rare event sampler for each distinct rare event. One of the main results proved in this paper is presented next, while complete technical assumptions on $P^{\theta}$ are given in Section 4 .

Main result: Sensitivity bounds and a sensitivity index for rare events. For $0<M<\infty$ let

$$
\overline{\mathcal{A}}_{M}=\left\{A: P^{\theta}(A)=e^{-M}\right\} .
$$

denote the sets, parametrized by the positive constant $M$, of all events which are equally probable (or rather equally rare if $M \gg 0)$. Then, for any $A \in \overline{\mathcal{A}}_{M}$ we have

$$
\mathcal{I}_{v,-}^{\theta}(M) \leq S_{v}^{\theta}(A) \leq \mathcal{I}_{v,+}^{\theta}(M)
$$

where

$$
\mathcal{I}_{v, \pm}^{\theta}(M):= \pm \inf _{\alpha>0}\left\{\frac{H_{v}^{\theta}( \pm \alpha)+M}{\alpha}\right\} \quad \text { with } \quad H_{v}^{\theta}(\alpha)=\log \mathbb{E}_{P^{\theta}}\left[e^{\alpha v^{T} W^{\theta}}\right] .
$$

(Note that $H_{v}^{\theta}(\alpha)$ is the cumulant generating function for the score function $W^{\theta}$ defined in (3).) Furthermore, denoting by $P_{\alpha}^{\theta}$ the exponential family of tilted measures

$$
\frac{d P_{\alpha}^{\theta}}{d P^{\theta}}=e^{\alpha v^{T} W^{\theta}-H_{v}^{\theta}(\alpha)}
$$

we have

$$
\mathcal{I}_{v, \pm}^{\theta}(M)=\left.\frac{d}{d \alpha} H_{v}^{\theta}(\alpha)\right|_{\alpha= \pm \alpha_{ \pm}}=\mathbb{E}_{P_{\alpha_{ \pm}}^{\theta}}\left[v^{T} W^{\theta}\right]
$$

Here $\alpha_{ \pm}$are determined by

$$
\mathcal{R}\left(P_{ \pm \alpha_{ \pm}}^{\theta} \| P^{\theta}\right)=M
$$

and $\mathcal{R}(Q \| P)$ denotes the relative entropy of $Q$ with respect to $P$. See Figure 1 for a graphical depiction of the characterization of $\alpha_{ \pm}$.

The proposed rare event sensitivity indices (7) are bounds for the gradient-based indices (1). They do not require a rare event sampler for each rare event $A$, as one readily sees in the definition of (7) or (8), and they apply to the entire class $\overline{\mathcal{A}}_{M}$ of rare events, that is for the probability level sets for the parametric model $P^{\theta}$. Intuitively, $\mathcal{I}_{v, \pm}^{\theta}(M)$ balances between the rarity of the event as quantified by $M$ and the cost to be paid in order to make the event less rare as quantified by $H_{v}^{\theta}(\alpha)$. Note also that, due to the monotonicity of (77) in $M$, the rare event sensitivity indices $\mathcal{I}_{v}^{\theta, \pm}(M)$ actually characterize the sensitivity of the model $P^{\theta}$ for each family

$$
\mathcal{A}_{M}:=\left\{A: P^{\theta}(A) \geq e^{-M}\right\},
$$

i.e., all events which are less rare than the threshold $e^{-M}$. In this sense, the bounds (6) present similar computational advantages and trade-offs as other sensitivity bounds for typical observables (not rare event dependent), such as the Cramér-Rao information bounds, see (5). Namely they are less accurate than the exact gradient-based indices (10), but they can be used to determine insensitive parameters and directions $v$, without requiring recalculation for different events A. We present a simple demonstration of such an insensitivity analysis based on the bounds (6) in the last example of Section 6 .

Additionally, in ordinary Cramer-Rao bounds, the sensitivity of the parametric model is encoded into the Fisher information matrix (the variance of the score function), for rare event the cumulant generating function of the score function plays a central role. Since the cumulant generating function controls rare events (as in Cramer's Theorem) our bounds show that the rare events associated to the score function control the sensitivity of all rare events. The question of the tightness of the sensitivity bounds will be addressed in [23], which discusses the UQ and sensitivity analysis for more general risk-sensitive functionals. 
For a practical implementation of the bound one can use concentration equalities [24] in a similar manner to UQ bounds for regular observables, 25, and obtain easily computable but less accurate bounds, see Section 5 . As the bound involves the moment generating function of the score function, the rare event simulation techniques mentioned at the beginning of the introduction could also in principle be used to solve the optimization problem in the sensitivity indices. We plan to revisit this issue in a follow-up publication.

The paper is organized as follows. In Section 2 we begin with the study of an optimization problem which appears several times throughout the paper and then proceed with the definition and the properties of the Rényi divergence. In Section 3 we derive our main UQ bounds based on the Rényi divergence optimized over its parameter. We then derive in Section 4 information inequalities for the sensitivity indices. In Section 5 we discuss the practical implementation of the sensitivity indices for rare events, via concentration inequalities or via numerical estimation. We illustrate our results on several distributions from the exponential family in Section 6, and in Section 7, we present sensitivity bounds for large deviation rate functions.

\section{Mathematical Preliminaries}

\subsection{An optimization problem}

In order to obtain optimal UQ bounds we have to consider a certain optimization problem involving cumulant generating functions. We note that a bound function with similar structure has been derived and studied recently in [26, 27, 28], and we slightly generalize and reformulate those results in this section.

Let $\mathcal{X}$ be a Polish space, $\mathcal{B}(\mathcal{X})$ the associated Borel $\sigma$-algebra and denote by $\mathcal{P}(\mathcal{X})$ the set of all probability measures on $(\mathcal{X}, \mathcal{B}(\mathcal{X}))$. Given a probability measure $P \in \mathcal{P}(\mathcal{X})$ and a measurable function $g: \mathcal{X} \rightarrow \mathbb{R}$ consider the moment generating function $\mathbb{E}_{P}\left[e^{\alpha g}\right]$ with $\alpha \in \mathbb{R}$. We will assume that $g$ is such that the moment generating function is finite in a neighborhood of the origin and denote the space of such functions by $\mathcal{E}$.

Definition 1. A measurable function $g: \mathcal{X} \rightarrow \mathbb{R}$ belongs to the set $\mathcal{E}$ if and only if there exists $\alpha_{0}>0$ such that $\mathbb{E}_{P}\left[e^{ \pm \alpha_{0} g}\right]<\infty$.

If $g \in \mathcal{E}$ then as is well known $g$ has finite moments of all orders, see also the discussion Appendix A,

Definition 2. Given $P \in \mathcal{P}(\mathcal{X})$ and $g \in \mathcal{E}$ the cumulant generating function of $g$ is given by

$$
H(\alpha):=\log \mathbb{E}_{P}\left[e^{\alpha g}\right] .
$$

A family of probability measures naturally associated to $H(\alpha)$ is the exponential family $P_{\alpha}$ given by

$$
\frac{d P_{\alpha}}{d P}=e^{\alpha g-H(\alpha)}
$$

which is well-defined if $H(\alpha)<\infty$.

In Appendix A, we summarize various useful properties of cumulant generating functions. These will be needed to study the following minimization problems, which arise in the definition of the sensitivity indices introduced in Section 1 .

Proposition 3. Let $P \in \mathcal{P}(\mathcal{X})$ and $g \in \mathcal{E}$ with $g$ not a constant $P$-a.s. Suppose $\left(d_{-}, d_{+}\right)$is the largest open set such that $H(\alpha)<\infty$ for all $\alpha \in\left(d_{-}, d_{+}\right)$.

1. For any $M \geq 0$ the optimization problems

$$
\inf _{\alpha>0} \frac{H( \pm \alpha)+M}{\alpha}
$$

have unique minimizers $\alpha^{ \pm} \in\left[0, \pm d_{ \pm}\right]$. Let $M_{ \pm}$be defined by

$$
M_{ \pm}=\lim _{\alpha \nearrow \pm d_{ \pm}} \pm \alpha H^{\prime}( \pm \alpha)-H( \pm \alpha)
$$

Then the minimizers $\alpha_{ \pm}=\alpha_{ \pm}(M)$ are finite for $M<M_{ \pm}$and $\alpha_{ \pm}(M)= \pm d_{ \pm}$if $M \geq M_{ \pm}$.

2. If $\alpha_{ \pm}(M)< \pm d_{ \pm}$then

$$
\frac{H\left( \pm \alpha_{ \pm}\right)+M}{\alpha_{ \pm}}=\inf _{\alpha>0} \frac{H( \pm \alpha)+M}{\alpha}= \pm H^{\prime}\left( \pm \alpha_{ \pm}\right)= \pm \mathbb{E}_{P_{ \pm \alpha_{ \pm}}}[g],
$$

where $\alpha_{ \pm}(M)$ is strictly increasing in $M$ and is determined by the equation

$$
\mathcal{R}\left(P_{ \pm \alpha_{ \pm}} \| P\right)=M .
$$


3. $M_{ \pm}$is finite in two distinct cases.

(a) If $\pm d_{ \pm}<\infty$ (in which case $g$ is unbounded above/below) $M_{ \pm}$is finite if $\lim _{\alpha \rightarrow \pm d_{ \pm}} H( \pm \alpha):=H\left(d_{ \pm}\right)<\infty$ and $\lim _{\alpha \rightarrow \pm d_{ \pm}} \pm H^{\prime}( \pm \alpha):= \pm H^{\prime}\left(d_{ \pm}\right)<\infty$, and for $M \geq M_{ \pm}$we have

$$
\inf _{\alpha>0} \frac{H( \pm \alpha)+M}{\alpha}=\frac{H\left(d_{ \pm}\right)+M}{ \pm d_{ \pm}}= \pm \mathbb{E}_{P_{d_{ \pm}}}[g]+\frac{M-M_{+}}{ \pm d_{ \pm}} .
$$

(b) If $\pm d_{ \pm}=\infty$ and $M_{ \pm}$is finite then $g$ is $P$-a.s. bounded above/below and for $M \geq M_{ \pm}$we have

$$
\inf _{\alpha>0} \frac{H( \pm \alpha)+M}{\alpha}=\operatorname{ess~sup}_{x \in \mathcal{X}}\{ \pm g(x)\} .
$$

Proof. The proof of Proposition 3 can be found in Appendix A. A geometric depiction of the Proposition when $M<M_{ \pm}$ is shown in Figure 1(a).

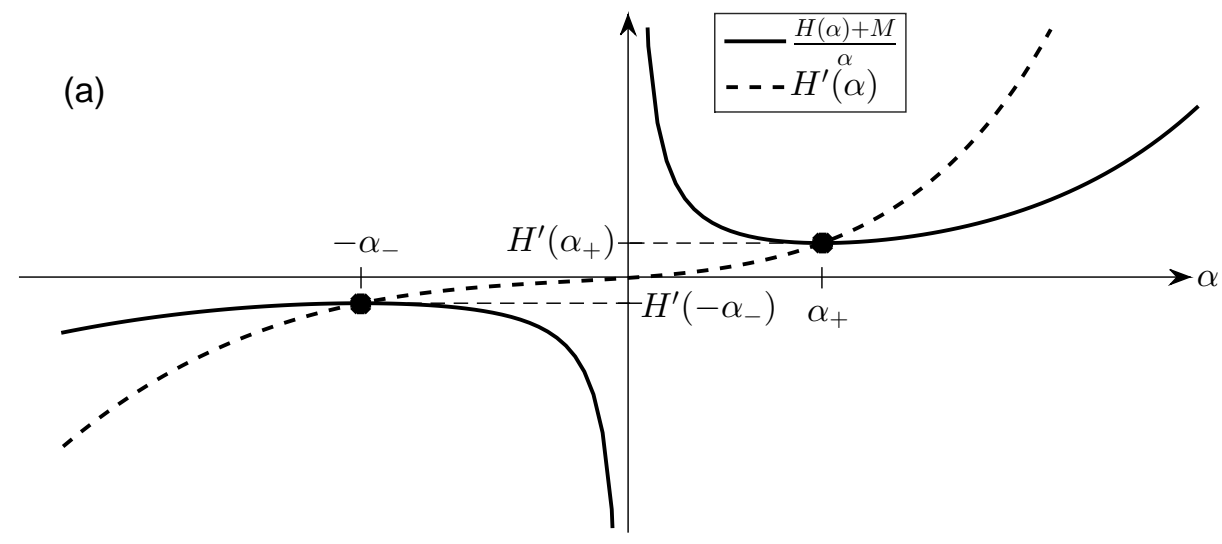

(b)

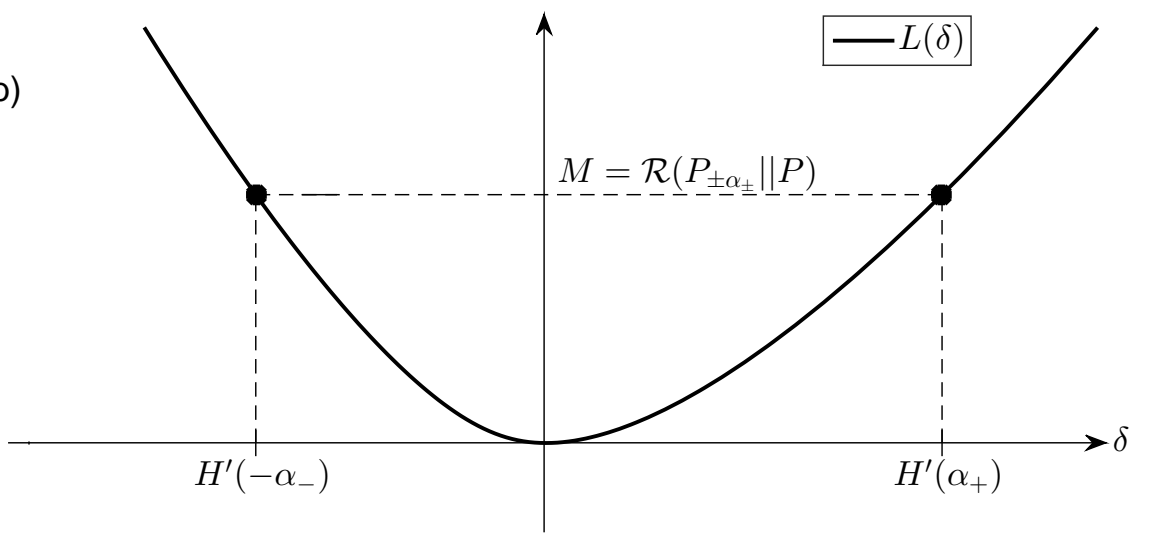

Figure 1: (a) Graphical representation of (9) which depicts the relation between the minimum of $(H(\alpha)+M) / \alpha$ (solid line) and the derivative of cumulant generating function, $H^{\prime}(\alpha)$ (dashed line). Using the fact that $\inf _{\alpha>0}(H(-\alpha)+M) / \alpha=$ $-\sup _{\alpha<0}(H(\alpha)+M) / \alpha$, we display the relation for $\alpha_{-}$at the third quadrant. Note that both minimizers are attained at the intersections of the two curves, however, the two branches are generally not symmetric resulting in different values (i.e., $\alpha_{+} \neq \alpha_{-}$). (b) Graphical representation of Remark 4 which relates the optimal values of $(H(\alpha)+M) / \alpha$ with the Legendre transform of the cumulant generating function. For demonstration clarity, we assume that $\mathbb{E}[g]=0$ so that $L(\delta) \geq 0$ and $L(\delta)=0$ if and only if $\delta=0$.

Unless the random variable $g$ is symmetric, in general the two optimization problems are not related to each other since they involve the cumulant generating function for $\alpha>0$ and $\alpha<0$ respectively.

Remark 4. An alternative characterization of the minimizers uses the Legendre-Fenchel transform of $H(\alpha)$,

$$
L(\delta)=\sup _{\alpha \in \mathbb{R}}\{\alpha \delta-H(\alpha)\}
$$


which is a convex function with a mimimum equal to 0 that is attained at $\mathbb{E}_{P}[g]$. The optimality condition (10) for $\alpha_{ \pm}$ can be written, equivalently, as

$$
M=L\left( \pm \delta_{ \pm}\right) \text {where } \delta_{ \pm}= \pm H^{\prime}\left( \pm \alpha_{ \pm}\right) \text {. }
$$

Thus the set of values of $M$ for which a finite minimizer exists corresponds to the possible level sets for $L(\delta)$ (the rate function in Cramer's theorem [29]), provided $L(\delta)$ is strictly convex.

If the function $g$ is centered, i.e., $\mathbb{E}_{P}[g]=0$, then when $M=0$ the minimizers are $\alpha_{ \pm}=0$ and we can expand $\alpha_{ \pm}$as a Taylor series in the variable $\sqrt{M}$ as the following proposition shows, which was proved in [28, Lemma 2.10] and will be useful for non-rare events.

Proposition 5 (Linearization). If $g \in \mathcal{E}$ with $\mathbb{E}_{P}[g]=0$ then we have

$$
\inf _{\alpha>0} \frac{H( \pm \alpha)+M}{\alpha}=\sqrt{2 \operatorname{Var}_{P}(g) M}+O(M)
$$

\subsection{Rényi divergence, relative entropy, and a variational principle}

In this section, we discuss the concepts of Rényi divergence and associated variational representations and duality formulas. These tools provide the mathematical foundations for the uncertainty quantification and sensitivity analysis methods introduced in the subsequent sections.

Given $P, Q \in \mathcal{P}(\mathcal{X})$, we pick a reference measure $R \in \mathcal{P}(\mathcal{X})$, such that $P \ll R$ and $Q \ll R$ (i.e., $P$ and $Q$ are absolutely continuous with respect to $R$ ). Denote by $p=\frac{d P}{d R}$ (resp. $q=\frac{d Q}{d R}$ ) the Radon-Nikodym derivative of $P$ (resp. $Q$ ) with respect to the reference measure $R$. Then, for $\alpha>0, \alpha \neq 1$, the Rényi divergence of degree $\alpha$ of $Q$ with respect to $P$ is defined by (cf. [30, 21])

$$
\mathcal{R}_{\alpha}(Q \| P):=\left\{\begin{array}{cl}
\frac{1}{\alpha(\alpha-1)} \log \int_{p q>0}\left(\frac{q}{p}\right)^{\alpha} d P & \text { if } Q \ll P \text { or } \alpha<1 \\
+\infty & \text { otherwise }
\end{array},\right.
$$

and is independent of the choice of the reference measure $R$. Another common definition of Rényi divergence utilizes the factor $\frac{1}{\alpha-1}$ (cf. [31, 32, 33]) instead of $\frac{1}{\alpha(\alpha-1)}$. When $P$ and $Q$ are mutually absolutely continuous, Rényi divergence can be written without reference to a reference measure as

$$
\mathcal{R}_{\alpha}(Q \| P)=\frac{1}{\alpha(\alpha-1)} \log \mathbb{E}_{P}\left[\left(\frac{d Q}{d P}\right)^{\alpha}\right]=\frac{1}{\alpha(\alpha-1)} \log \mathbb{E}_{P}\left[\exp \left\{\alpha \log \frac{d Q}{d P}\right\}\right],
$$

where the rightmost expression reveals that Rényi divergence is proportional to the cumulant (or log-moment) generating function for the logarithm of the Radon-Nikodym derivative (i.e., $\left.\log \frac{d Q}{d P}\right)$. The definition of Rényi divergence is extended to $\alpha=1$ by letting $\mathcal{R}_{\alpha}(Q \| P)=\mathcal{R}(Q \| P)$, where $\mathcal{R}(Q \| P)$ is the relative entropy (or Kullback-Leibler divergence) defined by

$$
\mathcal{R}(Q \| P):=\left\{\begin{array}{cc}
\int_{p q>0} \frac{q}{p} \log \frac{q}{p} d P & \text { if } Q \ll P \\
+\infty & \text { otherwise }
\end{array},\right.
$$

and we have $\lim _{\alpha \rightarrow 1} \mathcal{R}_{\alpha}(Q \| P)=\mathcal{R}(Q \| P)$. In view of (13) we can also extend $\mathcal{R}_{\alpha}(Q \| P)$ to negative values of $\alpha$ if $Q$ and $P$ are mutually absolutely continuous. Due to our convention for the definition of $\mathcal{R}_{\alpha}(Q \| P)$ we have the symmetry $\mathcal{R}_{\alpha}(Q \| P)=\mathcal{R}_{1-\alpha}(P \| Q)$ and thus, in particular, $\mathcal{R}_{0}(Q \| P)=\mathcal{R}(P \| Q)$. Further properties of the Rényi divergence can be found for example in $34,32,33$.

A variational formula in terms of Rényi divergence, recently derived in [26, Theorem 2.1], will play a central role in this paper.

Theorem 6 (Variational representation involving Rényi divergence). Let $\beta, \gamma \in \mathbb{R} \backslash\{0\}$ with $\beta<\gamma$ and let $P \in \mathcal{P}(\mathcal{X})$. For any bounded and measurable $f: \mathcal{X} \rightarrow \mathbb{R}$ we have

$$
\begin{aligned}
& \frac{1}{\beta} \log \mathbb{E}_{Q}\left[e^{\beta f}\right]=\inf _{P \in \mathcal{P}(\mathcal{X})}\left\{\frac{1}{\gamma} \log \mathbb{E}_{P}\left[e^{\gamma f}\right]+\frac{1}{\gamma-\beta} \mathcal{R}_{\frac{\gamma}{\gamma-\beta}}(Q \| P)\right\}, \\
& \frac{1}{\gamma} \log \mathbb{E}_{Q}\left[e^{\gamma f}\right]=\sup _{P \in \mathcal{P}(\mathcal{X})}\left\{\frac{1}{\beta} \log \mathbb{E}_{P}\left[e^{\beta f}\right]-\frac{1}{\gamma-\beta} \mathcal{R}_{\frac{\gamma}{\gamma-\beta}}(P \| Q)\right\} .
\end{aligned}
$$

Remark 7. The variational representation formula (15) is a generalization of the Donsker-Varadhan variational formula involving relative entropy (also know as the Gibbs variational principle) [35, 36]. Indeed taking $\gamma=1$ and $\beta \rightarrow 0$ in (15) we obtain the well-known formula

$$
\log \mathbb{E}_{Q}\left[e^{f}\right]=\sup _{P \in \mathcal{P}(\mathcal{X})}\left\{\mathbb{E}_{P}[f]-\mathcal{R}(P \| Q)\right\} .
$$

Note that the variational formula (16) serves as the basis of the UQ theory for typical events developed in [26, 28, 37, 25]. 


\section{UQ Bounds for Rare Events}

Let $P, Q \in \mathcal{P}(\mathcal{X})$ and $g: \mathcal{X} \rightarrow \mathbb{R}$ be a measurable function. It is convenient to think of $Q$ as the "true" probabilistic model and of $P$ as a "nominal" or "reference" model. By Theorem 6 equation (14), we have the upper bound

$$
\frac{1}{\beta} \log \mathbb{E}_{Q}\left[e^{\beta g}\right] \leq \frac{1}{\gamma} \log \mathbb{E}_{P}\left[e^{\gamma g}\right]+\frac{1}{\gamma-\beta} \mathcal{R} \frac{\gamma}{\gamma-\beta}(Q \| P),
$$

which constitutes an upper bound for risk-sensitive observables (i.e., where tail events matter). The upper bound consists of two terms; one being an estimate of the risk-sensitive observable under the "nominal" model and the second term being the cost to be paid for the substitution of the "true" model, here, quantified by the Rényi divergence.

By taking the limit $\beta \rightarrow 0$, it formally holds that

$$
\mathbb{E}_{Q}[g] \leq \frac{1}{\gamma} \log \mathbb{E}_{P}\left[e^{\gamma g}\right]+\frac{1}{\gamma} \mathcal{R}(Q \| P),
$$

which is an upper bound for typical (i.e., not risk-sensitive) observables. This upper bound (18) was the starting point for deriving UQ and sensitivity bounds for typical observables in 26, 27, 28, 37, 25. Here we derive respective bounds for log-probabilities of rare events, which form a particular class of risk-sensitive observables. In other words, we are interested in bounding quantities of the form $\log Q(A)-\log P(A)$. The following theorem summarizes the UQ bounds for rare events.

Theorem 8. (a) Fix $P, Q \in \mathcal{P}(\mathcal{X})$ and let $A \in \mathcal{B}(\mathcal{X})$ be such that $P(A)>0$ and $Q(A)>0$. Then

$$
\sup _{\alpha>0}\left\{-(\alpha+1) \mathcal{R}_{\alpha+1}(P \| Q)+\frac{1}{\alpha} \log P(A)\right\} \leq \log Q(A)-\log P(A) \leq \inf _{\alpha>0}\left\{\alpha \mathcal{R}_{\alpha+1}(Q \| P)-\frac{1}{\alpha+1} \log P(A)\right\} .
$$

(b) If $P$ and $Q$ are mutually absolutely continuous, then (19) can be rewritten as

$$
-\inf _{\alpha>0}\left\{\frac{\log \mathbb{E}_{P}\left[\left(\frac{d Q}{d P}\right)^{-\alpha}\right]-\log P(A)}{\alpha}\right\} \leq \log Q(A)-\log P(A) \leq \inf _{\alpha>1}\left\{\frac{\log \mathbb{E}_{P}\left[\left(\frac{d Q}{d P}\right)^{\alpha}\right]-\log P(A)}{\alpha}\right\} .
$$

Proof. (a) We first prove the upper bound. By setting $\beta=\alpha$ and $\gamma=\alpha+1$ in (17), we get

$$
\frac{1}{\alpha} \log \mathbb{E}_{Q}\left[e^{\alpha g}\right] \leq \frac{1}{\alpha+1} \log \mathbb{E}_{P}\left[e^{(\alpha+1) g}\right]+\mathcal{R}_{\alpha+1}(Q \| P) .
$$

By taking $\alpha>0$ and considering $g=0$ on $A, g=-M$ on $A^{c}$ and then sending $M \rightarrow \infty$, the above inequality becomes

$$
\frac{1}{\alpha} \log Q(A) \leq \frac{1}{\alpha+1} \log P(A)+\mathcal{R}_{\alpha+1}(Q \| P) .
$$

Multiplying with $\alpha$ and subtracting $\log P(A)$, we have

$$
\log Q(A)-\log P(A) \leq \alpha \mathcal{R}_{\alpha+1}(Q \| P)-\frac{1}{\alpha+1} \log P(A) .
$$

Since this holds for all $\alpha>0$, the upper bound in (19) is proved. For the lower bound, we reverse $Q$ and $P$ in (17) and proceed as in the upper bound.

(b) Substituting the Rényi formula (13) in (19), we get

$$
\sup _{\alpha>0}\left\{-\frac{1}{\alpha} \log \mathbb{E}_{Q}\left[\left(\frac{d P}{d Q}\right)^{\alpha+1}\right]+\frac{1}{\alpha} \log P(A)\right\} \leq \log Q(A)-\log P(A) \leq \inf _{\alpha>0}\left\{\frac{1}{\alpha+1} \log \mathbb{E}_{P}\left[\left(\frac{d Q}{d P}\right)^{\alpha+1}\right]-\frac{1}{\alpha+1} \log P(A)\right\} .
$$

Equivalently,

$$
\sup _{\alpha>0}\left\{-\frac{1}{\alpha} \log \mathbb{E}_{P}\left[\left(\frac{d P}{d Q}\right)^{\alpha}\right]+\frac{1}{\alpha} \log P(A)\right\} \leq \log Q(A)-\log P(A) \leq \inf _{\alpha>1}\left\{\frac{1}{\alpha} \log \mathbb{E}_{P}\left[\left(\frac{d Q}{d P}\right)^{\alpha}\right]-\frac{1}{\alpha} \log P(A)\right\} .
$$

which is exactly (20).

We turn next to determining the optimal $\alpha$ in (20) using the results from Section 2.1. We select the function $g=$ $\log \frac{d Q}{d P} \in \mathcal{E}$, whose cumulant generating function is

$$
H(\alpha)=\log \mathbb{E}_{P}\left[e^{\alpha \log \frac{d Q}{d P}}\right] .
$$

As is readily apparent from the bound (20), in order to obtain non-trivial upper and lower bounds we should assume $H(\alpha)$ is finite in an open neighborhood of the interval $[0,1]$. If we assume this then the function $H(\alpha)$ has the following elementary properties: 
1. $H(0)=H(1)=0$.

2. $H^{\prime}(0)=-\mathcal{R}(P \| Q), H^{\prime}(1)=\mathcal{R}(Q \| P)$.

3. More generally for any $\alpha$ we have

$$
H^{\prime}(\alpha)=\mathbb{E}_{P_{\alpha}}\left[\log \frac{d Q}{d P}\right]=\mathcal{R}\left(P_{\alpha} \| P\right)-\mathcal{R}\left(P_{\alpha} \| Q\right),
$$

where $P_{\alpha}$ is the exponential family given by

$$
\frac{d P_{\alpha}}{d R}=\frac{q^{\alpha} p^{1-\alpha}}{\int q^{\alpha} p^{1-\alpha} d R}
$$

The family $P_{\alpha}$ interpolates between $P$ and $Q$ since $P_{0}=P$ and $P_{1}=Q$.

Using these properties, as well as Proposition 3 we obtain the following, explicit UQ bounds for all rare events $A$ such that $-\log P(A)=M$.

Theorem 9 (UQ bounds for rare events). Let $P, Q \in \mathcal{P}(\mathcal{X})$ be mutually absolutely continuous and assume that $H(\alpha)$ given in (21) is finite for $\alpha$ in a neighborhood of $[0,1]$. Let $M_{ \pm}, 0<M_{ \pm} \leq \infty$, be the constants given in Proposition 3 (with $\left.g=\log \frac{d Q}{d P}\right)$. For any $A \in \mathcal{B}(\mathcal{X})$ with $P(A)=e^{-M}>0$ with $M \leq M_{ \pm}$we have

$$
-\mathcal{R}\left(P_{-\alpha_{-}} \| Q\right) \leq \log Q(A) \leq\left\{\begin{array}{cc}
0 & \text { if } M<\mathcal{R}(Q \| P) \\
-\mathcal{R}\left(P_{\alpha_{+}} \| Q\right) & \text { if } M \geq \mathcal{R}(Q \| P)
\end{array} .\right.
$$

where $\alpha_{ \pm}=\alpha_{ \pm}(M)$ are the (unique) solutions of

$$
\mathcal{R}\left(P_{ \pm} \alpha_{ \pm} \| P\right)=M=-\log P(A) .
$$

Proof. With $H(\alpha)$ given in (21) and setting $M=-\log P(A)$, part (b) of Theorem 8 is rewritten as

$$
-\inf _{\alpha>0}\left\{\frac{H(-\alpha)+M}{\alpha}\right\} \leq \log Q(A)+M \leq \inf _{\alpha>1}\left\{\frac{H(\alpha)+M}{\alpha}\right\} .
$$

The lower bound is then an immediate consequence of Proposition 3 together with (22) and (25).

However, the upper bound involves a modified calculation since the infimum is taken only over $\alpha>1$. We first note that the corresponding minimization of the quantity

$$
B(\alpha ; M)=\frac{H(\alpha)+M}{\alpha},
$$

arising also in the proof of Proposition 3, e.g. (62), separates into two distinct cases. Indeed, for any $\alpha>1$ we have

$$
B^{\prime}(\alpha ; M)=\frac{\alpha H^{\prime}(\alpha)-H(\alpha)-M}{\alpha^{2}}>\frac{1 \cdot H^{\prime}(1)-H(1)-M}{\alpha^{2}}=\frac{\mathcal{R}(Q \| P)-M}{\alpha^{2}},
$$

using the Properties 1-3 above for $H(\alpha)$ and the fact that $\alpha H^{\prime}(\alpha)-H(\alpha)$ is a strictly increasing function of $\alpha$ in $[0, \infty)$.

First, (28) implies that if

$$
\mathcal{R}(Q \| P)>M=-\log P(A),
$$

then $B(\alpha ; M)$ is strictly increasing in $\alpha$, hence the infimum on the upper bound of (26) occurs at $\alpha=1$. In this case, using that $H(1)=0$, we obtain that

$$
\inf _{\alpha>1}\left\{\frac{H(\alpha)+M}{\alpha}\right\}=M
$$

On the other hand, if we have

$$
\mathcal{R}(Q \| P) \leq M=-\log P(A),
$$

then $B(\alpha ; M)$ has a unique minimum, and the minimizer (equivalently, the minimizer of the upper bound of (26) ) occurs at the unique finite root $\alpha_{+}=\alpha_{+}(M)>1$, namely

$$
\inf _{\alpha>1}\left\{\frac{H(\alpha)+M}{\alpha}\right\}=H^{\prime}\left(\alpha_{+}\right)=M-\mathcal{R}\left(P_{\alpha_{+}} \| Q\right)
$$

We now combine (30) and (32) with the upper bound of (26) to obtain (24).

Note that the upper bound in (24) is not discontinuous in $M$ since for $M=\mathcal{R}(P \| Q), \alpha_{+}=1$ and by Property 3 we have that $P_{1}=Q$; thus $\mathcal{R}\left(P_{\alpha_{+}} \| Q\right)=\mathcal{R}(Q \| Q)=0$. 
Remark 10. We obtain the trivial bound $\log Q(A) \leq 0$ in Theorem 9 when the true measure $Q$ is (relatively) too far from the reference model $P$. This relative "distance" (see (29) and (31)) is quantified by the ratio $\frac{\mathcal{R}(Q \| P)}{-\log P(A)}$ which is required to be less than 1 to obtain an informative upper bound in (24).

Remark 11. We can interpret the condition for $\alpha_{ \pm}$in (25) by noting that the measure $P$ conditioned on the rare event $A, P_{\mid A}$ satisfies $\mathcal{R}\left(P_{\mid A} \| P\right)=-\log P(A)$. Theorem 9 states that one should find the proper mixtures of $P$ and $Q$ (as described by $\left.P_{ \pm \alpha_{ \pm}}\right)$so that $\mathcal{R}\left(P_{ \pm \alpha_{ \pm}} \| P\right)=\mathcal{R}\left(P_{\mid A} \| P\right)$. The bounds for $-\log Q(A)$ are then simply $\mathcal{R}\left(P_{ \pm \alpha_{ \pm}} \| Q\right)$.

Finally, we note that a much cruder UQ bound than (19) can be obtained by considering the upper lower bounds obtained by taking $\alpha=\infty$ in (19). Indeed, we can consider the alternative definition of Rényi divergence [33]

$$
\mathcal{D}_{\alpha}(Q \| P)=\alpha \mathcal{R}_{\alpha}(Q \| P),
$$

and accordingly rewrite (19) for $\mathcal{D}_{\alpha}(Q \| P)$. Noting that

$$
\mathcal{D}_{\infty}(Q \| P)=\sup _{x \in \mathcal{X}} \log \frac{d Q}{d P},
$$

also referred as worst-case regret, [33], we can bound (19) from above and below by selecting $\alpha=\infty$. This substitution obviously yields a less sharp version of (19), namely the (trivial) bound

$$
\inf _{x \in \mathcal{X}} \log \frac{d Q}{d P}=-\mathcal{D}_{\infty}(P \| Q) \leq \log Q(A)-\log P(A) \leq \mathcal{D}_{\infty}(Q \| P)=\sup _{x \in \mathcal{X}} \log \frac{d Q}{d P}
$$

Note that this bound is valid if $M_{ \pm}<\infty$ and $M>M_{ \pm}$, as long as $\log \frac{d Q}{d P}$ is bounded from above/below.

\section{Sensitivity Indices for Rare Events}

In this section we consider a parametric family of probability measures $P^{\theta} \in \mathcal{P}(\mathcal{X})$ with $\theta \in \mathbb{R}^{K}$ and we assume $P^{\theta} \ll R$, where $R$ is a reference measure in $P(\mathcal{X})$ and with density $p^{\theta}=\frac{d P^{\theta}}{d R}$. We further assume that the mapping $\theta \mapsto p^{\theta}(x)$ is twice differentiable with respect to $\theta$ for all $x \in \mathcal{X}$ together with a suitable integrability condition on $\log p^{\theta}(x)$ to allow the interchange of integral and derivatives. The sensitivity index for a rare event $A \in \mathcal{B}(\mathcal{X})($ with $P(A)>0)$ in the direction $v \in \mathbb{R}^{K}$ is then given by

$$
S_{v}^{\theta}(A):=\lim _{\epsilon \rightarrow 0} \frac{\log P^{\theta+\epsilon v}(A)-\log P^{\theta}(A)}{\epsilon}=\mathbb{E}_{P_{\mid A}^{\theta}}\left[v^{T} W^{\theta}\right]=\frac{\mathbb{E}_{P^{\theta}}\left[\chi_{A} v^{T} W^{\theta}\right]}{P(A)},
$$

where $W^{\theta}=\nabla_{\theta} \log p^{\theta}$ is the score of the probability measure $P^{\theta}$, see also (44).

Here, we derive computationally tractable bounds on the sensitivity indices $S_{v}^{\theta}(A)$ and corresponding new rare event sensitivity indices $\mathcal{I}_{v, \pm}^{\theta}$, starting from the UQ bounds presented in Section 3 .

By considering the measures $Q=P^{\theta+\epsilon v}$ and $P=P^{\theta}$ we have that $\log \frac{d P^{\theta+\epsilon v}}{d P^{\theta}}=O(\epsilon)$ and thus it is natural to rescale the parameter $\alpha$ according to

$$
\alpha=\frac{\alpha_{0}}{\epsilon} .
$$

Taking $\epsilon \rightarrow 0$ we obtain a non-trivial bound for the sensitivity indices as the following Theorem shows. Next, in order to state our results we require the cumulant generating function for the score function $W^{\theta}$ defined in (3):

$$
H_{v}^{\theta}(\alpha)=\log \mathbb{E}_{P^{\theta}}\left[e^{\alpha v^{T} W^{\theta}}\right] .
$$

This cumulant generating function has the following elementary properties:

1. $\left(H_{v}^{\theta}\right)^{\prime}(0)=\mathbb{E}_{P^{\theta}}\left[v^{T} W^{\theta}\right]=0$.

2. $\left(H_{v}^{\theta}\right)^{\prime \prime}(0)=v^{T} \mathbb{E}_{P^{\theta}}\left[W^{\theta}\left(W^{\theta}\right)^{T}\right] v=v^{T} \mathcal{F}\left(P^{\theta}\right) v$ where $\mathcal{F}\left(P^{\theta}\right)$ denotes the Fisher information matrix for the parametric family $P^{\theta}$.

3. More generally we have

$$
\left(H_{v}^{\theta}\right)^{\prime}(\alpha)=\mathbb{E}_{P_{\alpha}^{\theta}}\left[v^{T} W^{\theta}\right]
$$

where $P_{\alpha}^{\theta}$ is the exponential family with

$$
\frac{d P_{\alpha}^{\theta}}{d P^{\theta}}=e^{\alpha v^{T} W^{\theta}-H_{v}^{\theta}(\alpha)} .
$$


Theorem 12. Assume that the mapping $\theta \mapsto p^{\theta}(x)$ is $\mathcal{C}^{2}$ for all $x \in \mathcal{X}$ and that for each $\alpha_{0}>0$ there exists $\delta>0$ such that

$$
\mathbb{E}_{P^{\theta}}\left[e^{\alpha_{0} v^{T} \nabla_{\theta} \log p^{\theta}+\alpha_{0} \frac{\delta}{2} \sup _{\left|\theta-\theta^{\prime}\right|<\delta}\left|v^{T} \nabla_{\theta}^{2} \log p^{\theta^{\prime}} v\right|}\right]<\infty
$$

Then

$$
-\inf _{\alpha>0}\left\{\frac{H_{v}^{\theta}(-\alpha)-\log P^{\theta}(A)}{\alpha}\right\} \leq S_{v}^{\theta}(A) \leq \inf _{\alpha>0}\left\{\frac{H_{v}^{\theta}(\alpha)-\log P^{\theta}(A)}{\alpha}\right\} .
$$

Proof. Rewriting (20) for $P^{\theta+\epsilon v}$ and $P^{\theta}$ and substituting $\alpha$ from (35), we get the upper bound

$$
\log P^{\theta+\epsilon v}(A)-\log P^{\theta}(A) \leq\left\{\frac{\log \mathbb{E}_{P^{\theta}}\left[e^{\frac{\alpha_{0}}{\epsilon}\left(\log p^{\theta+\epsilon v}-\log p^{\theta}\right)}\right]-\log P^{\theta}(A)}{\alpha_{0} / \epsilon}\right\}
$$

valid for all $\alpha_{0}>\epsilon$. Dividing by $\epsilon$, sending $\epsilon \rightarrow 0$, and then infimizing on $\alpha_{0}$, we get

$$
\lim _{\epsilon \rightarrow 0} \frac{1}{\epsilon}\left(\log P^{\theta+\epsilon v}(A)-\log P^{\theta}(A)\right) \leq \inf _{\alpha_{0}>0}\left\{\frac{\lim { }_{\epsilon \rightarrow 0} \log \mathbb{E}_{P^{\theta}}\left[e^{\frac{\alpha_{0}}{\epsilon}\left(\log p^{\theta+\epsilon v}-\log p^{\theta}\right)}\right]-\log P^{\theta}(A)}{\alpha_{0}}\right\}
$$

In order to complete the proof of the upper bound we have to interchange between the limit and the integral. This is justified by the dominated convergence theorem since we have from Taylor's theorem that

$$
e^{\alpha_{0} \frac{1}{\epsilon}\left(\log p^{\theta+\epsilon v}-\log p^{\theta}\right)}=e^{\alpha_{0}\left(v^{T} \nabla_{\theta} \log p^{\theta}+\frac{\epsilon}{2} v^{T} \nabla_{\theta}^{2} \log p^{\theta^{\prime}} v\right)} \leq e^{\alpha_{0}\left(v^{T} \nabla_{\theta} \log p^{\theta}+\frac{\delta}{2} \sup _{\left|\theta-\theta^{\prime}\right|<\delta}\left|v^{T} \nabla_{\theta}^{2} \log p^{\theta^{\prime}} v\right|\right)}
$$

for some $\theta^{\prime}$ in the interval defined by the points $\theta$ and $\theta+\epsilon v$ and for $\epsilon<\delta$. Therefore,

$$
S_{v}^{\theta}(A) \leq \inf _{\alpha_{0}>0} \frac{\log \mathbb{E}_{P^{\theta}}\left[e^{\alpha_{0} v^{T} \nabla_{\theta} \log p^{\theta}}\right]-\log P^{\theta}(A)}{\alpha_{0}}
$$

which establishes the upper bound in (36). The lower bound in (36) is proved similarly.

Using (9) and (10) from Proposition 3 to evaluate the infimum in (36), we obtain a representation of the bounds.

Theorem 13 (Sensitivity indices for rare events). Under the same assumptions as in Theorem 12 consider the family

$$
\mathcal{A}_{M}=\left\{A: P^{\theta}(A) \geq e^{-M}\right\}
$$

of all events $A$ which are less rare than a specified threshold $e^{-M}$. Then there exists $M_{ \pm}$such that for $M<M_{ \pm}$and any $A \in \mathcal{A}_{M}$ we have

$$
\mathcal{I}_{v,-}^{\theta}(M) \leq S_{v}^{\theta}(A) \leq \mathcal{I}_{v,+}^{\theta}(M)
$$

where

$$
\mathcal{I}_{v, \pm}^{\theta}(M):= \pm \inf _{\alpha>0}\left\{\frac{H_{v}^{\theta}( \pm \alpha)+M}{\alpha}\right\}=\mathbb{E}_{P_{ \pm \alpha_{ \pm}}^{\theta}}\left[v^{T} W^{\theta}\right]
$$

and $\alpha_{ \pm}$are determined by

$$
\mathcal{R}\left(P_{ \pm \alpha_{ \pm}}^{\theta} \| P^{\theta}\right)=M
$$

Similarly to Theorem 13 the rare event sensitivity indices $\mathcal{I}_{v, \pm}^{\theta}(M)$ characterize the sensitivity of the model $P^{\theta}$ for each $M$-level set $\overline{\mathcal{A}}_{M}:=\left\{A: \log P^{\theta}(A)=-M\right\}$, i.e. corresponding to all events which are equally rare and characterized by $M$.

The new sensitivity indices defined in (38) are in general less sharp than the gradient-based indices $S_{v}^{\theta}(A)$ in (34), due to the inequalities (37). However, they do not require a rare event sampler for each rare event $A$, as one readily sees by comparing (40) to (38). In fact the indices $\mathcal{I}_{v, \pm}^{\theta}(M)$ are identical for the entire classes of rare events in $\mathcal{A}_{M}$ or in $\overline{\mathcal{A}}_{M}$. In this sense, they present similar computational advantages and trade-offs as other sensitivity indices for typical observables (not rare event-dependent), such as Fisher information bounds, [28; in particular, they are less sharp but can be used to efficiently screen out insensitive parameters in directions $v$ in parameter space, i.e directions $v$ where $\mathcal{I}_{v, \pm}^{\theta}(M)=\mathbb{E}_{P_{ \pm \alpha_{ \pm}}^{\theta}}\left[v^{T} W^{\theta}\right] \approx 0$; we refer for such sensitivity screening results to [38], at least for typical events and observables. 


\section{Bounds and Approximations for the Rare Event Sensitivity Indices}

The upper and lower bounds in Theorem 12 and the representation of the sensitivity indices in Theorem[13] suggest at least two approaches to practically implement the indices $\mathcal{I}_{v, \pm}^{\theta}(M)$. The first one is based on concentration inequalities, while the second one relies on the direct statistical and numerical estimation of the indices $\mathcal{I}_{v, \pm}^{\theta}(M)$. We next discuss the first approach.

Concentration Inequalities. Concentration inequalities, i.e., explicit bounds of the probability of tail events, are often obtained via a Chernoff bound by using computable upper bounds on the cumulant generating function of the random variable. Such upper bounds typically involve only a few features of the underlying random variable such as mean, variance, bounds, higher moments, and so on, see for instance [24].

Here we can naturally use such inequalities to provide simplified and computable bounds for the variational formula for the sensitivity index, namely

$$
\mathcal{I}_{v, \pm}^{\theta}(M):= \pm \inf _{\alpha>0}\left\{\frac{H_{v}^{\theta}( \pm \alpha)+M}{\alpha}\right\}
$$

by bounding the cumulant generating function of the score function $H_{v}^{\theta}(\alpha)=\log \mathbb{E}_{P^{\theta}}\left[e^{\alpha v^{T} W^{\theta}}\right]$. We provide two such examples, by making the assumption that the score function is bounded. One can prove similar results in the same spirit by using different assumptions on the tail behavior of the score function, e.g. if we assume that the score $v^{T} W^{\theta}$ is a sub-Gaussian or a sub-Poissonian random variable [24].

A similar use of various concentration inequalities in order to obtain computable uncertainty quantification bounds for ordinary observables was proposed recently in [25.

Theorem 14 (Bernstein sensitivity bounds for rare events). We consider the same assumptions as in Theorem 12; we further assume

$$
\sup _{x \in \mathcal{X}} v^{T} W^{\theta}(x) \leq b_{v}
$$

for some $b_{v}>0$. Furthermore, let

$$
\mathcal{F}\left(P^{\theta}\right)=\mathbb{E}_{P^{\theta}}\left[W^{\theta}\left(W^{\theta}\right)^{T}\right]
$$

be the Fisher information matrix for the parametric family $P^{\theta}$. Then, we have the following two bounds on the cumulant generating function of the score function (3), $H_{v}^{\theta}(\alpha)$, and the sensitivity index $\mathcal{I}_{v,+}^{\theta}(M)$ :

(a) For all $\alpha>0$ we have the concentration inequality:

$$
H_{v}^{\theta}(\alpha)=\log \mathbb{E}_{P^{\theta}}\left[e^{\alpha v^{T} W^{\theta}}\right] \leq \frac{v^{T} \mathcal{F}\left(P^{\theta}\right) v}{b_{v}^{2}} \phi\left(b_{v} \alpha\right),
$$

where $\phi(x)=e^{x}-x-1$.

(b) Using the notation of Theorem 13, we have for all $M<M_{ \pm}$:

$$
\mathcal{I}_{v,+}^{\theta}(M) \leq b_{v} M+\sqrt{2 v^{T} \mathcal{F}\left(P^{\theta}\right) v M} .
$$

Proof. (a) Follows immediately from Theorem 2.9 in [24] by noting that $\mathbb{E}_{P^{\theta}} W^{\theta}=0$, and therefore we have that $\operatorname{Var}_{P^{\theta}}\left(v^{T} W^{\theta}\right)=v^{T} \mathbb{E}_{P^{\theta}}\left[W^{\theta}\left(W^{\theta}\right)^{T}\right] v=v^{T} \mathcal{F}\left(P^{\theta}\right) v$.

(b) First we note that $\phi(x) \leq \frac{x^{2}}{2(1-x)}$, for $0 \leq x<1$. Therefore, part (a) implies that

$$
\mathcal{I}_{v,+}^{\theta}(M):=\inf _{\alpha>0}\left\{\frac{H_{v}^{\theta}(\alpha)+M}{\alpha}\right\} \leq \inf _{0<b_{v} \alpha<1}\left\{v^{T} \mathcal{F}\left(P^{\theta}\right) v \frac{\alpha^{2}}{2\left(1-b_{v} \alpha\right)}+\frac{M}{\alpha}\right\} .
$$

We next change variables to $c=\left(b_{v} \alpha\right)^{-1}>1$, and then it is easy to show that the minimum occurs at $c^{*}=1+$ $b^{-1} \sqrt{\frac{v^{T} \mathcal{F}\left(P^{\theta}\right) v}{2 M}}$. We conclude by substituting into the right hand side of (41).

Remark 15 (A Bennett sensitivity bound for rare events). A much tighter concentration inequality than (39) is given by the Bennett inequality in 29], Lemma 2.4.1; see also Figure 2. Here, using again that $\mathbb{E}_{P^{\theta}} W^{\theta}=0$ and $\operatorname{Var}_{P^{\theta}}\left(v^{T} W^{\theta}\right)=$ $v^{T} \mathcal{F}\left(P^{\theta}\right) v$, we have that

$$
H_{v}^{\theta}(\alpha)=\log \mathbb{E}_{P^{\theta}}\left[e^{\alpha v^{T} W^{\theta}}\right] \leq \log \left(\frac{b_{v}{ }^{2}}{b_{v}{ }^{2}+\sigma_{v}^{2}} \exp \left(-\alpha \sigma_{v}^{2} / b_{v}\right)+\frac{\sigma_{v}^{2}}{b_{v}{ }^{2}+\sigma_{v}^{2}} \exp \left(\alpha b_{v}\right)\right)
$$


for all $\alpha \geq 0$ and where $\sigma_{v}^{2}$ is any upper bound of $\operatorname{Var}_{P^{\theta}}\left(v^{T} W^{\theta}\right)=v^{T} \mathcal{F}\left(P^{\theta}\right) v$. Therefore, we can pick

$$
\sigma_{v}^{2}:=v^{T} \mathcal{F}\left(P^{\theta}\right) v \quad \text { and } \quad b_{v}:=\sup _{x \in \mathcal{X}} v^{T} W^{\theta}(x) .
$$

Then, the corresponding bound on $\mathcal{I}_{v,+}^{\theta}(M)$ is not analytically tractable, however the resulting variational representation is one dimensional and is trivial to find the optimal solution numerically:

$$
\mathcal{I}_{v,+}^{\theta}(M) \leq \inf _{\alpha>0}\left\{\frac{1}{\alpha} \log \left(\frac{b_{v}{ }^{2}}{{b_{v}}^{2}+\sigma_{v}^{2}} \exp \left(-\alpha \sigma_{v}^{2} / b_{v}\right)+\frac{\sigma_{v}^{2}}{b_{v}{ }^{2}+\sigma_{v}^{2}} \exp \left(\alpha b_{v}\right)\right)+\frac{M}{\alpha}\right\} .
$$

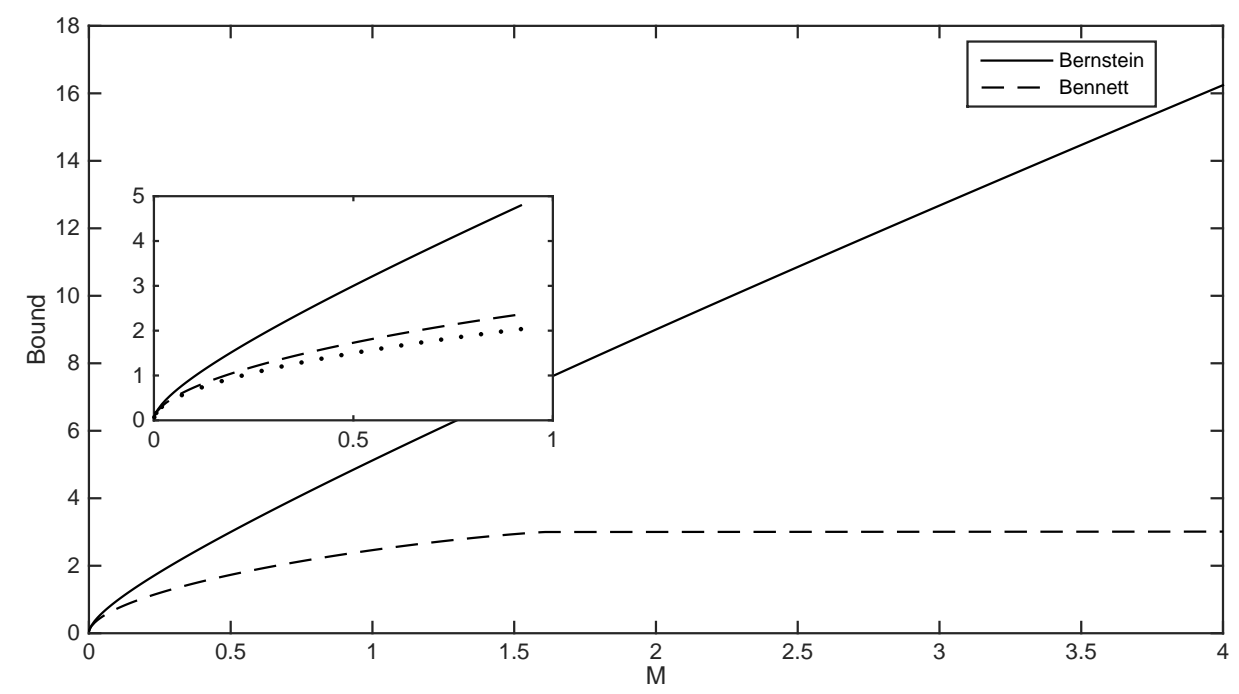

Figure 2: Bernstein (solid) (40) and Bennett (dashed) (43) concentration inequality bounds for fixed values of $b_{v}$ and $\sigma_{v}$. The Bennett inequality is sharper and in fact it is also tight at $M=\infty$ where its asymptotic limit is $b_{v} \geq \mathbb{E}_{P_{ \pm \alpha_{ \pm}}^{\theta}}\left[v^{T} W^{\theta}\right]$. The inset plot zooms in near the origin where the event is not so rare and the bounds have a behaviour close to the linearized bound (dots) (45).

Remark 16 (Linearized bounds). If an event $A$ is not rare, i.e. $P^{\theta}(A) \approx 1$ or $-\log P^{\theta}(A) \approx 0$, the calculation of the sensitivity indices $\mathcal{I}_{v, \pm}^{\theta}(M)$ is fairly trivial. Indeed, we can expand the sensitivity index (38) in a power series in $\sqrt{-\log P^{\theta}(A)}$, if we assume that

$$
M=-\log P^{\theta}(A) \ll 1 .
$$

Then the sensitivity index in the bound (37) is approximated as

$$
\mathcal{I}_{v, \pm}^{\theta}(M)= \pm \sqrt{2 v^{T} \mathcal{F}\left(P^{\theta}\right) v M}+O(M),
$$

where $\mathcal{F}\left(P^{\theta}\right)$ is again the Fisher information matrix for the parametric family $P^{\theta}$. The expansion (45) follows from the observation that $\mathbb{E}_{P^{\theta}}\left[\nabla_{\theta} \log p^{\theta}\right]=0$ and a direct application of Proposition 5 together with the formula for $\left(H_{v}^{\theta}\right)^{\prime \prime}(0)=$ $v^{T} \mathcal{F}\left(P^{\theta}\right) v$. We also note that the Fisher information matrix arises when sensitivity bounds for ordinary observables are linearized [28, Theorem 2.14]. However, in order to get the bound in (45) two linearizations were performed here; one capturing the closeness between $P^{\theta+\epsilon v}$ and $P^{\theta}$ and another capturing that the event is actually not rare. Interestingly, the linearization (45) is also identical to the dominant term in the Bernstein bound (40) for small $M=-\log P^{\theta}(A)$, i.e. when

$$
M<\frac{2 v^{T} \mathcal{F}\left(P^{\theta}\right) v}{b_{v}}
$$

Remark 17. Although the bounds (40) and (43) are less tight than the index $\mathcal{I}_{v,+}^{\theta}(M)$, they are much easier to estimate than $\mathcal{I}_{v,+}^{\theta}(M)$ since they only involve the sampling of the Fisher information matrix $\mathcal{F}\left(P^{\theta}\right)$, whose calculation does not entail rare event sampling. Finally, the sensitivity bounds (40) and (43) hold for any $M$ and they are not just asymptotically true in $M$, unlike the linearization (45) that requires (44), and in addition has an uncontrolled higher order error term $O(M)$. 
Direct statistical estimation methods for $\mathcal{I}_{v, \pm}^{\theta}(M)$. The two representations of the sensitivity indices (38) in Theorem [13, either as a variational problem, or using the Kullback-Leibler divergence suggest at least two approaches to estimate the indices $\mathcal{I}_{v, \pm}^{\theta}(M)$ using direct numerical simulation.

First, since the optimization problem in the variational representation in (38) is one dimensional, it is fairly trivial to solve numerically, hence the main roadblock is the estimation of the cumulant generating function $H_{v}^{\theta}( \pm \alpha)$. Existing numerical methods to tackle either one of these problems already exist in the literature. For instance, for the calculation of moment and cumulant generation functions can be performed using interacting particle systems methods [8, 9] or splitting techniques [10, 11, 12, 13].

Using the alternative representation (38) of the minimizer $\mathcal{I}_{v, \pm}^{\theta}(M)$, namely,

$$
\mathcal{I}_{v, \pm}^{\theta}(M)=\mathbb{E}_{P_{ \pm \alpha_{ \pm}}^{\theta}}\left[v^{T} W^{\theta}\right]
$$

demonstrates the need to sample from the tilted measures $P_{ \pm \alpha_{ \pm}}^{\theta}$, which in turn is also intimately related problem to estimating the cumulant generating function $H_{v}^{\theta}( \pm \alpha)$. When $\pm \alpha_{ \pm}$is fairly close to zero, the sampling distribution $P_{ \pm \alpha_{ \pm}}^{\theta}$ in (46) is a perturbation of $P^{\theta}$; in this case the Free Energy Perturbation method, see [39] Section 2.4.1, can be used to simulate efficiently $\mathcal{I}_{v, \pm}^{\theta}(M)$. However, by Proposition $3 \alpha_{ \pm}(M)$ is increasing in $M$ and thus when $M=-\log P^{\theta}(A)$ grows, $\alpha_{ \pm}$in (46) can be large, see also the examples in Figure 2. In this case $P_{ \pm \alpha_{ \pm}}^{\theta}$ in (46) is not necessarily a perturbation of $P^{\theta}$. Multilevel Monte Carlo techniques such as chaining methods, see Section 11.6 in [40, or Thermodynamic Integration, as in Section 3.1 in [39, or the RESTART method [13] could in principle be used in the calculation of the indices (38).

These issues are outside of the scope of this paper and we plan to return to this topic and related implementations of the sensitivity indices in a follow-up publication.

\section{Examples}

Exponential family of distributions. It is instructive to consider the example of exponential families, that is, the family of measures with densities $p^{\theta}(x)$ given by

$$
p^{\theta}(x)=e^{\theta^{T} t(x)-F(\theta)} .
$$

where $t(x)$ is the vector of sufficient statistics and $F(\theta)=\log \mathbb{E}_{R}\left[e^{\theta^{T} t}\right]$. The score function is then given by

$$
W^{\theta}(x)=\nabla_{\theta} \log p^{\theta}(x)=t(x)-\nabla F(\theta)=t(x)-\mathbb{E}_{P^{\theta}}[t],
$$

and the cumulant generating function is given by

$$
\begin{aligned}
H_{v}^{\theta}(\alpha): & =\log \mathbb{E}_{P^{\theta}}\left[\exp \left\{\alpha v^{T} \nabla \log p^{\theta}\right\}\right] \\
& =\log \int \exp \left\{\alpha v^{T}(t(x)-\nabla F(\theta))\right\} \exp \left\{\theta^{T} t(x)-F(\theta)\right\} R(d x) \\
& =\log \int \exp \left\{(\alpha v+\theta)^{T} t(x)-\left(F(\theta)+\alpha v^{T} \nabla F(\theta)\right)\right\} R(d x) \\
& =F(\alpha v+\theta)-F(\theta)-\alpha v^{T} \mathbb{E}_{P^{\theta}}[t]=F(\alpha v+\theta)-F(\theta)-\alpha v^{T} \nabla F(\theta) .
\end{aligned}
$$

It is worth noting that the cumulant generating function is the Bregman divergence, 33, associated with $F$ at points $\alpha v+\theta$ and $\theta$. This is an explicit quantification of the cost to be paid for tilting the distribution in order to make the event less rare. Additionally, the tilted measure $P_{\alpha}^{\theta}$ has density

$$
\frac{d P_{\alpha}^{\theta}}{d R}=\exp \left\{\alpha v^{T}\left(t(x)-\mathbb{E}_{P^{\theta}}[t]\right)-H_{v}^{\theta}(\alpha)+\theta^{T} t(x)-F(\theta)\right\}=\exp \left\{(\theta+\alpha v)^{T} t(x)-F(\theta+\alpha v)\right\}=p^{\theta+\alpha v}
$$

and thus $P_{\alpha}^{\theta}=P^{\theta+\alpha v}$ also belongs to the same exponential family. Finally the optimal $\alpha_{ \pm}$are the solutions of the equation

$$
\log P(A)=-\mathcal{R}\left(P^{\theta+\alpha v} \| P^{\theta}\right)=F(\theta+\alpha v)-F(\theta)+\alpha v^{T} \nabla F(\theta+\alpha v)
$$

and the sensitivity bounds and corresponding indices $\mathcal{I}_{v, \pm}^{\theta}(M)$ in Theorem 13 take the form

$$
\mathcal{I}_{v,-}^{\theta}(M)=\mathbb{E}_{P^{\theta-\alpha_{-} v}}\left[v^{T} t\right]-\mathbb{E}_{P^{\theta}}\left[v^{T} t\right] \leq S_{v}^{\theta}(A) \leq \mathbb{E}_{P^{\theta+\alpha_{+} v}}\left[v^{T} t\right]-\mathbb{E}_{P^{\theta}}\left[v^{T} t\right]=\mathcal{I}_{v,+}^{\theta}(M),
$$

i.e., they are expressed as the difference between the mean sufficient statistics under the optimally tilted distributions and the mean sufficient statistics under the original distribution. 
Finally, many exponential families have explicit formulas for the cumulant generating function of the sufficient statistic. Thus, the sensitivity bounds as well as the two characterizations of the optimal values $\alpha_{ \pm}$can be visualized. Figures $3(\mathrm{a})$ 3(d) show the upper bound function, $(H(\alpha)+M) / \alpha$, with $H(\alpha):=H_{v}^{\theta}(\alpha)$ as well as the derivative of the cumulant generating function for various distributions and various values of $M$. The minimizer of the upper bound, $\alpha_{+}$, can be geometrically characterized as the intersection of the upper bound function and $H^{\prime}(\alpha)$. Similar plots are obtained for the lower bound.

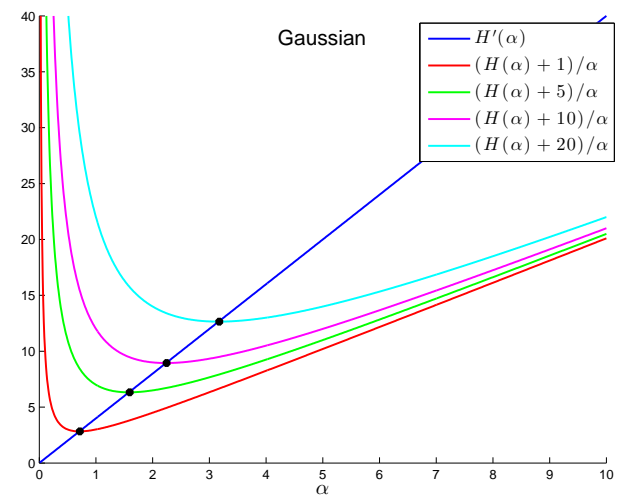

(a) Gaussian distribution with $\theta$ being the mean value over the variance. The density of Gaussian distribution is given by $p^{\theta}(x)=e^{\theta x-F(\theta)}$ where $F(\theta)=\frac{1}{2} \sigma^{2} \theta^{2}$ with $\sigma^{2}$ being the variance.

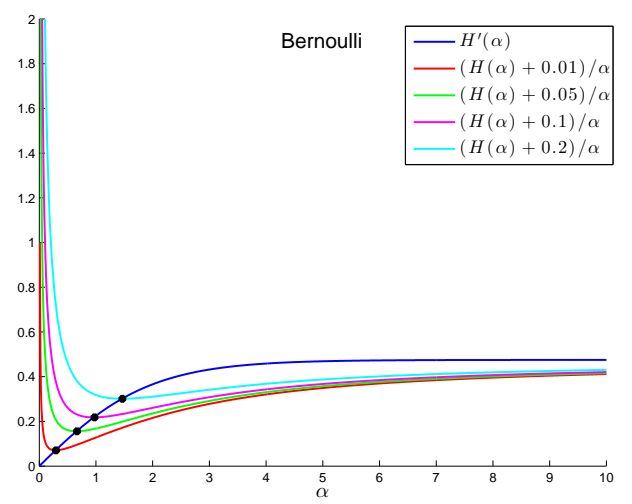

(c) Bernoulli distribution with $\theta$ being the logarithm of the ratio $\frac{p}{1-p}$ where $p$ is the probability of success. The density of Bernoulli distribution is given by $p^{\theta}(x)=e^{\theta x-F(\theta)}$ where $F(\theta)=\log \left(1+e^{\theta}\right)$.

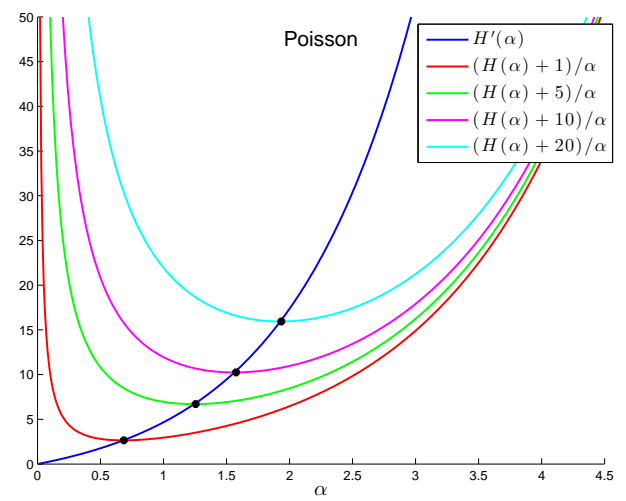

(b) Poisson distribution with $\theta$ being the logarithm of the Poisson rate. The density of Poisson distribution is given by $p^{\theta}(x)=e^{\theta x-F(\theta)}$ where $F(\theta)=e^{\theta}$.

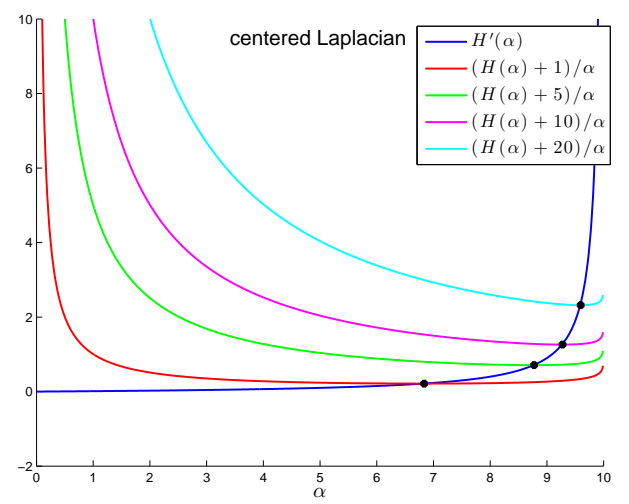

(d) Centered Laplacian distribution with $\theta$ being the negative of the mean value. The density of centered Laplacian distribution is given by $p^{\theta}(x)=e^{\theta|x|-F(\theta)}$ where $F(\theta)=\log \left(-\frac{1}{\theta}\right)$.

Figure 3: Graphical representation of the upper bound function, $(H(\alpha)+M) / \alpha$, and the derivative of cumulant generating function, $H^{\prime}(\alpha)$, for different members of the exponential family of distributions and various values of $M$. We assume the positive direction (i.e., $v=1$ ) thus $H(\alpha):=H_{v}^{\theta}(\alpha)=F(\theta+\alpha)-F(\theta)-\alpha F^{\prime}(\theta)$. The minimizer of the upper bound, $\alpha_{+}=\alpha_{+}(M)$, is depicted as a black dot. Despite having a unique minimum, the shape of the bound function varies significantly across the distributions.

Normal distribution. We next consider the specific case of a normal distribution and demonstrate that the sensitivity bounds are easier to implement than the sampling of the rare event and the corresponding likelihood ratio method for rare events, discussed in Section 1. In this case we can consider the tail events such like $A=\{X>L\}$ or any other rare event $A$ characterized by the parameter $M$,

$$
M \geq-\log P^{\theta}(A), \quad \text { where } \quad P^{\theta}=\mathcal{N}\left(\mu, \sigma^{2}\right),
$$

as well as the corresponding sensitivity index (4):

$$
S_{v}^{\theta}(A)=\mathbb{E}_{P_{\mid A}^{\theta}} v^{T} W^{\theta}=\mathbb{E}_{P_{\mid A}^{\theta}}\left[v^{T} t(x)\right]-\mathbb{E}_{P^{\theta}}\left[v^{T} t\right] .
$$


On the other hand, we consider the bounds (47), where the optimal values of $\pm \alpha_{ \pm}$are given by the explicitely solvable equation

$$
\mathcal{R}\left(P_{ \pm \alpha_{ \pm}}^{\theta} \| P^{\theta}\right)=M
$$

In fact, using the notation of the exponential family we have that

$$
P^{\theta}=e^{\theta^{T} t(x)-F(\theta)}=\mathcal{N}\left(\mu, \sigma^{2}\right), \quad \text { where } t(x)=\left(x, x^{2} / 2\right), \quad \theta=\left(\theta_{1}, \theta_{2}\right)=\left(\mu / \sigma^{2},-\sigma^{-2}\right) .
$$

Furthermore, for any unit vector $v=\left(v_{1}, v_{2}\right)$,

$$
\left.W^{\theta}(x)=\left(x-\mu, x^{2} / 2-\left(\sigma^{2}+\mu^{2}\right) / 2\right), \text { and } \quad v^{T} W^{\theta}(x)=v_{1}(x-\mu)+v_{2}\left(x^{2} / 2-\left(\sigma^{2}+\mu^{2}\right) / 2\right)\right) .
$$

Next we define the corresponding tilted distributions

$$
P_{\alpha}^{\theta}=P^{\theta+\alpha v}=\mathcal{N}\left(\frac{\mu+\alpha \sigma^{2} v_{1}}{1-\alpha \sigma^{2} v_{2}}, \frac{\sigma^{2}}{1-\alpha \sigma^{2} v_{2}}\right) .
$$

Using the normality of the distributions $P_{\alpha}^{\theta}$ and $P^{\theta}$ we have:

$$
\mathcal{R}\left(P_{\alpha}^{\theta} \| P^{\theta}\right)=\frac{1}{2}\left[\frac{1}{1-\alpha \sigma^{2} v_{2}}-1+\log \left(1-\alpha \sigma^{2} v_{2}\right)+\alpha^{2} \sigma^{2}\left(\frac{v_{1}+\mu v_{2}}{1-\alpha \sigma^{2} v_{2}}\right)\right] .
$$

For instance, we can consider the solution of (49) in the case $v=(1,0)$. Then we obtain that $\alpha_{ \pm}=\sqrt{2 M+1} / \sigma$ and by replacing it in (50) we have the tilted distributions for upper and lower bounds in (47)

$$
P_{ \pm \alpha_{ \pm}}^{\theta}=\mathcal{N}\left(\mu \pm \sigma \sqrt{2 M+1}, \sigma^{2}\right)
$$

Furthermore the bounds and the corresponding sensitivity indices in (47)-where $S_{v}^{\theta}(A)$ is given by (48)-take the following simple form:

$$
\mathcal{I}_{v, \pm}^{\theta}(M)= \pm \sigma \sqrt{2 M+1} .
$$

It is worth noting that the upper and lower bounds $\mathcal{I}_{v, \pm}^{\theta}(M)$ do not involve the sampling of each specific rare event $A$ as required by (48).

Finally, this example provides a demonstration of a parameter insensitivity analysis based on the upper and lower bounds $\mathcal{I}_{v, \pm}^{\theta}(M)$ in Theorem[13, Specifically, if $\sigma \sqrt{2 M+1} \ll 1$ in (51), then we readily obtain from Theorem 13 that the probability of the rare event $A$ is insensitive with respect to the parameter $\theta_{1}=\mu / \sigma^{2}$. This insensitivity is quantified by the indices in (51) without having to calculate explicitly the gradient of $\log P^{\theta}(A)$ in the direction $v=(1,0)$.

\section{Sensitivity Bounds for Large Deviation Rate Functions}

Rare events are closely related to the theory of large deviations, [35, 29, and in this section we show how our sensitivity bounds and the sensitivity indices $\mathcal{I}_{v, \pm}^{\theta}(M)$ in Theorem 13 are related to large deviation rate functions. We first present a general framework which applies to any large deviation principle, and subsequently we discuss more concrete problems, such as independent, identically distributed (IID) and Markov sequences.

General result. Recall that a sequence of probability measures $\left\{P_{n}\right\}$ on a Polish space $\mathcal{X}$ satisfies a large deviation principle if there exists a lower-semicontinuous function $I: \mathcal{X} \rightarrow \mathbb{R}$ with compact level sets such that for any Borel set $A \subset \mathcal{X}$

$$
-\underline{I}(A) \leq \liminf _{n \rightarrow \infty} \frac{1}{n} \log P_{n}(A) \leq \limsup _{n \rightarrow \infty} \frac{1}{n} \log P_{n}(A) \leq-\bar{I}(A),
$$

where, with $A^{\circ}$ denoting the interior of $A$ and $\bar{A}$ the closure of $A$,

$$
\underline{I}(A)=\inf _{x \in A^{\circ}} I(x), \quad \bar{I}(A)=\inf _{x \in \bar{A}} I(x) .
$$

For "nice sets", e.g. open and convex, we have that $\underline{I}(A)=\bar{I}(A)$ in which case the set $A$ is called an $I$-continuity set, 29], although we do not need such an assumption here.

To perform the sensitivity analysis introduced in previous sections, we will consider a parametric family $P_{n}^{\theta}$ of probability measures satisfying a large deviation principle with rate function $I^{\theta}$; we also fix an event of interest denoted by $A$. We intend to obtain bounds on the relative rate of change of the probability of the rare event $A$, which in view of the large deviation principle (52) translates to bounds on the derivatives of the functions $\bar{I}^{\theta}(A)$ and $\underline{I}^{\theta}(A)$ with respect 
to $\theta$. We assume that $P_{n}^{\theta}$ has a density $p_{n}^{\theta}$ with respect to some reference measure $R(d x)$; we consider the score function $W_{n}^{\theta}=\nabla_{\theta} \log p_{n}^{\theta}(x)$, as well as the sensitivity indices

$$
S_{v, n}^{\theta}(A)=v^{T} \nabla_{\theta} \log P_{n}^{\theta}(A),
$$

which we assume both to be well-defined. Under the additional assumptions in Section 4 , see Theorem 12 and Theorem 13 . we obtain the following sensitivity bounds

$$
\mathcal{I}_{v, n,-}^{\theta}(M) \leq-\inf _{\alpha>0}\left\{\frac{H_{v, n}^{\theta}(-\alpha)-\log P_{n}^{\theta}(A)}{\alpha}\right\} \leq S_{v, n}^{\theta}(A) \leq \inf _{\alpha>0}\left\{\frac{H_{v, n}^{\theta}(\alpha)-\log P_{n}^{\theta}(A)}{\alpha}\right\} \leq \mathcal{I}_{v, n,+}^{\theta}(M),
$$

for all events $A$ such that $M \geq-\log P_{n}^{\theta}(A)$, where

$$
H_{v, n}^{\theta}(\alpha)=\log \mathbb{E}_{P_{n}^{\theta}}\left[e^{\alpha v^{T} W_{n}^{\theta}}\right] .
$$

All quantities are indexed by $n$ to denote their dependence on the sequence of the probability measures $\left\{P_{n}\right\}$. Using (52) and (54) we obtain immediately the following result.

Theorem 18 (Sensitivity indices and large deviation limits). Assume that the limit

$$
h_{v}^{\theta}(\alpha)=\lim _{n \rightarrow \infty} \frac{1}{n} H_{v, n}^{\theta}(\alpha)
$$

exists and define the rare event sensitivity indices

$$
\bar{s}_{v}^{\theta}(A):=\limsup _{n \rightarrow \infty} \frac{1}{n} S_{v, n}^{\theta}(A), \quad \underline{s}_{v}^{\theta}(A):=\liminf _{n \rightarrow \infty} \frac{1}{n} S_{v, n}^{\theta}(A) .
$$

Then we have

$$
-\inf _{\alpha>0}\left\{\frac{h_{v}^{\theta}(-\alpha)+\bar{I}^{\theta}(A)}{\alpha}\right\} \leq \underline{s}_{v}^{\theta}(A) \leq \bar{s}_{v}^{\theta}(A) \leq \inf _{\alpha>0}\left\{\frac{h_{v}^{\theta}(\alpha)+\underline{I}^{\theta}(A)}{\alpha}\right\} .
$$

Furthermore, as in Theorem 13, we can define the sensitivity indices in terms of $M$-level sets of the large deviation functionals (52):

$$
\mathcal{I}_{v, \infty,-}^{\theta}(M):=-\inf _{\alpha>0}\left\{\frac{h_{v}^{\theta}(-\alpha)+M}{\alpha}\right\}, \quad \mathcal{I}_{v, \infty,+}^{\theta}(M):=\inf _{\alpha>0}\left\{\frac{h_{v}^{\theta}(\alpha)+M}{\alpha}\right\} .
$$

In order to obtain more precise and concrete results and representations of the sensitivity indices we consider next some standard examples from the theory of large deviations.

Sequences of IID random variables. For IID sequences one can, unsurprisingly, bound the sensitivity of the large deviation rate function in terms of the moment generating function of the score function.

Theorem 19 (Sensitivity indices for IID random sequences). Let $X$ be a random vector taking values in $\mathbb{R}^{d}$ with probability distribution $P^{\theta}$ and density $p^{\theta}=\frac{d P^{\theta}}{d R}$ with respect to some reference measure $R$. Assume that the score function $W^{\theta}=$ $\nabla_{\theta} \log p^{\theta}$ satisfies the integrability conditions of Theorem 10 with cumulant generating function $H_{v}^{\theta}(\alpha)=\log \mathbb{E}_{P^{\theta}}\left[e^{\alpha v^{T} W^{\theta}}\right]$. Assume that the moment generating function $\mathbb{E}_{P^{\theta}}\left[e^{\lambda^{T} X}\right]$ is finite for $\lambda \in \mathbb{R}^{d}$ in the neighbourhood of the origin so that, if $X_{i}, i=1,2, \cdots$ is a sequence of IID random variables with common distribution $P^{\theta}$, then $S_{n}=\frac{1}{n} \sum_{k=1}^{n} X_{i}$ satisfies a large deviation principle with rate function

$$
I^{\theta}(x)=\sup _{\lambda \in \mathbb{R}^{d}}\left\{\lambda^{T} x-\log \mathbb{E}_{P^{\theta}}\left[e^{\lambda^{T} X}\right]\right\} .
$$

The sensitivity indices defined in (55) then satisfy

$$
-\inf _{\alpha>0}\left\{\frac{H_{v}^{\theta}(-\alpha)+\bar{I}^{\theta}(A)}{\alpha}\right\} \leq \underline{s}_{v}^{\theta}(A) \leq \bar{s}_{v}^{\theta}(A) \leq \inf _{\alpha>0}\left\{\frac{H_{v}^{\theta}(\alpha)+\underline{I}^{\theta}(A)}{\alpha}\right\} .
$$

Moreover, if $\underline{I}^{\theta}(A)<M_{+}\left(\bar{I}^{\theta}(A)<M_{-}\right)$, where $M_{ \pm}$are as in Proposition 3, then there exist finite $\alpha_{ \pm}$such that

$$
\mathbb{E}_{P_{-\alpha_{-}}^{\theta}}\left[v^{T} W^{\theta}\right] \leq \underline{s}_{v}^{\theta}(A) \leq \bar{s}_{v}^{\theta}(A) \leq \mathbb{E}_{P_{\alpha_{+}}^{\theta}}\left[v^{T} W^{\theta}\right]
$$

with

$$
\mathcal{R}\left(P_{-\alpha_{-}}^{\theta} \| P^{\theta}\right)=\bar{I}^{\theta}(A), \quad \mathcal{R}\left(P_{\alpha_{+}}^{\theta} \| P^{\theta}\right)=\underline{I}^{\theta}(A),
$$

and $P_{\alpha}^{\theta}$ is the measure with density $\frac{d P_{\alpha}^{\theta}}{d P^{\theta}}=e^{\alpha v^{T} W^{\theta}-H_{v}^{\theta}(\alpha)}$. Finally we can define sensitivity indices $\mathcal{I}_{v, \infty, \pm}^{\theta}$ similarly to (56). 
Proof. Let $Q_{n}$ denote the distribution of $\frac{S_{n}}{n}$ and $P_{n}^{\theta}=P^{\theta} \times \cdots \times P^{\theta}$ the joint distribution of $\left(X_{1}, \cdots, X_{n}\right)$ which has density $p_{n}^{\theta}\left(x_{1}, \cdots, x_{n}\right)=\prod_{1=1}^{n} p^{\theta}\left(x_{i}\right)$ with respect to $R_{n}=R \times \cdots \times R$. We have then

$$
\nabla_{\theta} \log Q_{n}(A)=\nabla_{\theta} \log P_{n}^{\theta}\left\{\left(x_{1}, \cdots, x_{n}\right): \frac{1}{n} \sum_{i=1}^{n} x_{i} \in A\right\} .
$$

The score function for the probability $P_{n}^{\theta}$ is given by

$$
W_{n}^{\theta}\left(x_{1}, \cdots, x_{n}\right)=\nabla_{\theta} \log p_{n}^{\theta}\left(x_{1}, \cdots, x_{n}\right)=\sum_{k=1}^{n} W^{\theta}\left(x_{k}\right)
$$

and thus we obtain

$$
\frac{1}{n} \log \mathbb{E}_{P_{n}^{\theta}}\left[e^{\alpha v^{T} W_{n}^{\theta}\left(X_{1}, \cdots, X_{n}\right)}\right]=\frac{1}{n} \log \mathbb{E}_{P_{n}^{\theta}}\left[\prod_{k=1}^{n} e^{\alpha v^{T} W^{\theta}\left(X_{k}\right)}\right]=\log \mathbb{E}_{P^{\theta}}\left[e^{\alpha v^{T} W^{\theta}(X)}\right] .
$$

Using Theorem 12 and taking $n \rightarrow \infty$ we obtain (57). Finally, using Proposition 3 we obtain the representation (58) and (59).

Markov sequences. We can apply our results to any stochastic processes for which a large deviation principle holds, but here we concentrate on the simplest case of discrete-time Markov chains (DTMC) with finite state space where the rate function is easy to obtain and we can dispense with technical assumptions. Let $\left\{X_{k}\right\}$ be an irreducible finite-state Markov chain with state space $\Sigma$ and transition matrix $\pi^{\theta}(i, j)$ depending on a parameter vector $\theta \in \mathbb{R}^{k}$. We assume that $\pi^{\theta}$ generates an ergodic Markov chain, that $\pi^{\theta}(i, j)$ depends smoothly on $\theta$ and that for any $i \in \Sigma$ the transition probabilities $\pi^{\theta}(i, j)$ and $\pi^{\theta+\epsilon v}(i, j)$ are mutually absolutely continuous for $\epsilon$ in a neighborhood of 0 and for all $v \in \mathbb{R}^{k}$ with $\|v\|=1$. We then define the score function

$$
W^{\theta}(i, j)=\nabla_{\theta} \log \pi^{\theta}(i, j),
$$

if $\pi^{\theta}(i, j)>0$, and set it equal to 0 otherwise. We assume, for simplicity, that the Markov chain starts in the (arbitrary) state $x_{0}$. The joint probability distribution of the Markov chain on the time interval from 0 to $n$ is

$$
P_{n}^{\theta}\left(x_{1}, \ldots, x_{n}\right)=\pi^{\theta}\left(x_{0}, x_{1}\right) \times \cdots \times \pi^{\theta}\left(x_{n-1}, x_{n}\right) .
$$

For any $f: \Sigma \rightarrow \mathbb{R}$, the sequence of random variables $S_{n}=\frac{1}{n} \sum_{k=1}^{n} f\left(X_{k}\right)$ satisfies a large deviation principle with a rate function which can be identified in terms of relative entropy [35, Theorem 8.4.3] and the contraction principle for large deviations [35, Theorem 1.3.2]. Let $l(i), i \in \Sigma$ denote a probability measure on the state space $\Sigma$ and let $\bar{\pi}(i, j)$ denote a transition kernel on $\Sigma$. For $\beta \in \mathbb{R}$ define

$$
I^{\theta}(\beta)=\inf _{\bar{\pi}, l}\left\{\sum_{i \in \Sigma} R\left(\bar{\pi}(i, \cdot) \| \pi^{\theta}(i, \cdot)\right) l(i): \sum_{i \in \Sigma} f(i) l(i)=\beta, \sum_{j \in \Sigma} l(j) \bar{\pi}(j, i)=l(i) \text { for } i \in \Sigma\right\} .
$$

The second constraint in this definition implies that $l$ is invariant under $\bar{\pi}$, while the first constraint enforces that the mean of $f$ under $l$ is $\beta$. The rate function $I^{\theta}=I^{\theta}(\beta)$ is then the minimum of a relative entropy cost for "tilting" from $\pi$ to $\bar{\pi}$, so that the mean of $f$ under the stationary distribution of $\bar{\pi}$ is equal to $\beta$. An alternative representation of $I^{\theta}$ is as the Legendre transform of the $\log \lambda^{\theta}(\alpha)$, where $\lambda^{\theta}(\alpha)$ is the maximal eigenvalue of the positive matrix $\pi^{\theta}(i, j) e^{\alpha f(j)},[29$.

Now we can directly deduce from Theorem 18 the following result on rare event sensitivity bounds for Markov sequences.

Theorem 20 (Sensitivity indices for Markov sequences). Let $\left\{X_{k}\right\}_{k=1,2, \cdots}$ be a ergodic Markov chain on the finite state space $\Sigma$ with transition probabilities $\pi^{\theta}(i, j)$. Assume that the transition probabilities $\pi^{\theta}$ depend smoothly on $\theta$ and that for all $i \in \Sigma, \pi^{\theta}(i, j)$ and $\pi^{\theta+\epsilon v}(i, j)$ are mutually absolutely continuous for $\epsilon$ sufficiently small and $\|v\|=1$. For $f: \Sigma \rightarrow \mathbb{R}$ the sequence $S_{n}=\frac{1}{n} \sum_{k=1}^{n} f\left(X_{k}\right)$ satisfies a large deviation principle with rate function $I^{\theta}(\beta)$ given in (60) and we have the sensitivity bounds

$$
-\inf _{\alpha>0}\left\{\frac{h_{v}^{\theta}(-\alpha)+\bar{I}^{\theta}(A)}{\alpha}\right\} \leq \underline{s}_{v}^{\theta}(A) \leq \bar{s}_{v}^{\theta}(A) \leq \inf _{\alpha>0}\left\{\frac{h_{v}^{\theta}(\alpha)+\underline{I}^{\theta}(A)}{\alpha}\right\}
$$

where

$$
h_{v}^{\theta}(\alpha)=\lim _{n \rightarrow \infty} \frac{1}{n} \mathbb{E}_{P_{n}^{\theta}}\left[e^{\alpha v^{T} \sum_{k=1}^{n} W^{\theta}\left(X_{k-1}, X_{k}\right)}\right] .
$$


We can represent $h_{v}^{\theta}(\alpha)$ as the logarithm of the maximal eigenvalue of the matrix $p^{\theta}(i, j) e^{\alpha v^{T} W^{\theta}(i, j)}$. Alternatively, consider the empirical measure for pairs $\left(X_{i-1}, X_{i}\right)$, which has the rate function

$$
J(l \otimes \bar{\pi})=\sum_{i \in \Sigma} R\left(\bar{\pi}(i, \cdot) \| \pi^{\theta}(i, \cdot)\right) l(i)
$$

whenever $l$ is stationary under $\bar{\pi}$ and $[l \otimes \bar{\pi}](i, j)=l(i) \bar{\pi}(i, j)$ ( $J$ is infinity otherwise). Using this rate function, we can evaluate $h_{v}^{\theta}(\alpha)$, since by Varadhan's lemma we have

$$
\begin{aligned}
& \lim _{n \rightarrow \infty} \frac{1}{n} \log \mathbb{E}_{P_{n}^{\theta}} e^{\alpha v^{T} \sum_{i=1}^{n} W^{\theta}\left(X_{i-1}, X_{i}\right)} \\
& \quad=\sup \left\{\alpha v^{T} \sum_{i, j \in \Sigma} W^{\theta}(i, j) l(i) \bar{\pi}(i, j)-\sum_{i \in \Sigma} R(\bar{\pi}(i, \cdot) \| \pi(i, \cdot)) l(i): \sum_{j \in \Sigma} l(j) \bar{\pi}(j, i)=l(i) \text { for } i \in \Sigma\right\} .
\end{aligned}
$$

Similarly to (60), this last variational problem is easily solved as a constrained convex programming problem. We demonstrate such an implementation in the next example.

Indeed, as a concrete example to illustrate the previous results we consider a Markov chain with five states with values $-2, \ldots, 2$. The transition probabilities are defined as

$$
\text { -2) } \underset{1-p_{-1}}{\stackrel{1}{\rightleftarrows}}\left(-1 \underset{1-p_{0}}{\stackrel{p_{-1}}{\rightleftarrows}}(0) \underset{1-p_{1}}{\stackrel{p_{0}}{\rightleftarrows}}(1) \underset{1}{\stackrel{p_{1}}{\rightleftarrows}}(2 .\right.
$$

Let $\theta=\left[p_{-1}, p_{0}, p_{1}\right]^{T}$ be the parameter vector and let $\left\{X_{i}\right\}$ be a Markov chain created from the above transition laws, and let $S_{n}$ be the empirical average of $X_{1}, \ldots, X_{n}$. We study the tightness of the sensitivity bound for the event $A=\left\{S_{n}=z\right\}$. Although $A$ is not an $I^{\theta}$-continuity set, using the special lattice structure of the set of states in the support of the empirical measure, we can easily show that $\lim _{n \rightarrow \infty} \frac{1}{n} \log P_{n}^{\theta}\left(S_{n}=z_{n}\right)=-I^{\theta}(z)$, as long as $z_{n} \rightarrow z$, when each $z_{n}$ is of the form $i / n$. The rate functional $I^{\theta}(z)$ is finite in the interval $[-1.5,1.5]$ and infinite elsewhere due to the fact that the transitions from state 2 (resp. -2) cannot happen more than $n / 2$ times making $\frac{1}{n} \sum_{i=1}^{n} x_{i} \in[-1.5,1.5]$.

In Figure 4 we present the sensitivity indices and the associated sensitivity bounds for various values of $z$ and various perturbation vectors $v$. Similarly, in Figure 5 we depict the sensitivity indices and the associated sensitivity bounds for various values of the parameter vector. Both figures suggest that the sensitivity bounds are informative and tightly follow the true values of the gradient sensitivity indices (55), which in the present setting coincide.
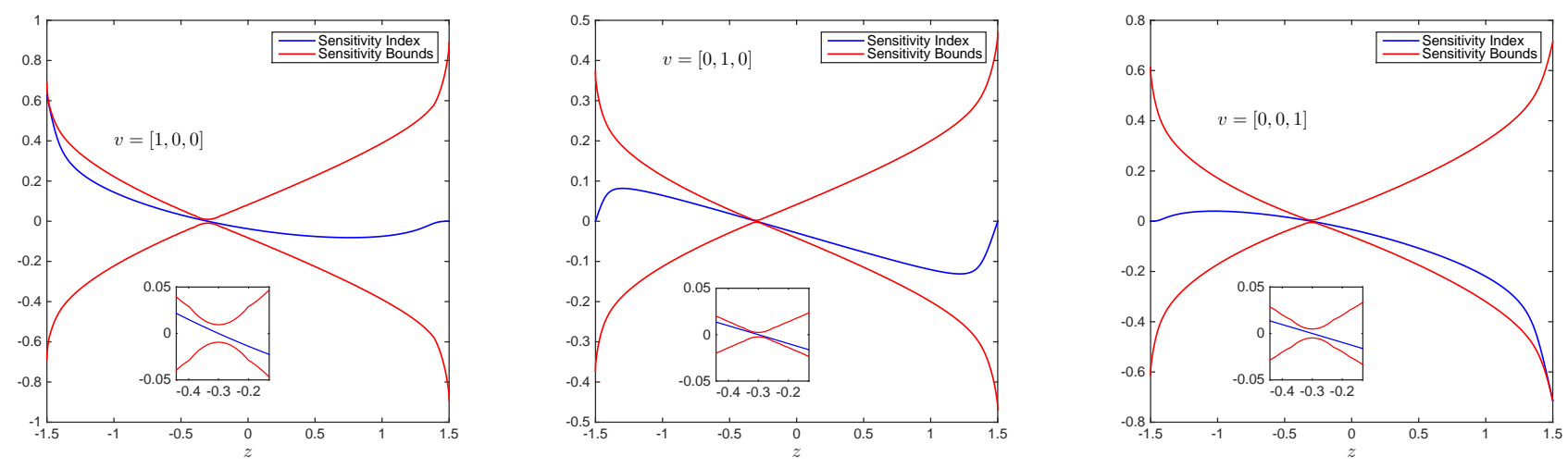

Figure 4: Sensitivity index (55) (blue) and the associated sensitivity bounds in (61) (red) for various levels of rare event probability as quantified by the variation in the values of $z$. Each panel corresponds to a different perturbation vector $v$. From left to right the perturbation vectors are the three orthonormal unit vectors. Parameter vector is kept fixed at $\theta=[0.2,0.5,0.7]^{T}$. Inset plots zoom around the zero value for the sensitivity index.

\section{A Appendix: Proposition and proofs}

Let $g \in \mathcal{E}$ (see Definition 11) and denote by $g_{ \pm}= \pm \operatorname{essup}\{ \pm g(x)\}$ its upper/lower bound (we allow the value $+\infty$ ). Recall that $H(\alpha)=\log \mathbb{E}_{P}\left[e^{\alpha g}\right]$ is the moment generating function with Legendre transform $L(\delta)=\sup _{\alpha \in \mathbb{R}}\{\alpha \delta-H(\alpha)\}$ and that $P_{\alpha}$ is the exponential family with $\frac{d P_{\alpha}}{d P}=e^{\alpha g-H(\alpha)}$. 

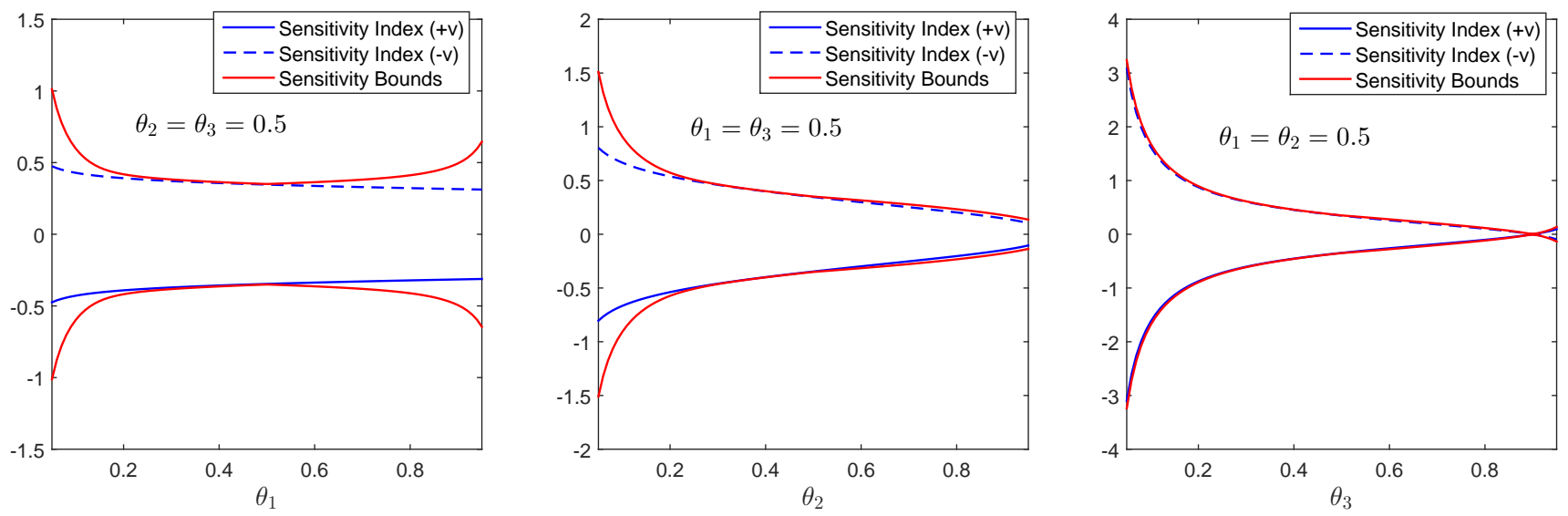

Figure 5: Sensitivity index for $v$ and $-v$ and the associated sensitivity bounds at different parameter regimes. Both the rare event and the perturbation vector are kept fixed at $x=1$ and $v=\frac{1}{\sqrt{3}}[1,1,1]^{T}$. In each panel, one of the parameters is varying while the other two are kept constant. Evidently, sensitivity bounds tightly follow the sensitivity indices in this demonstration.

Proposition 21. For $g \in \mathcal{E}$ we have

(a) The map $H(\alpha)$ is a convex function of $\alpha$, is finite in an interval $\left(d_{-}, d_{+}\right)$with $d_{-}<0<d_{+}$and $H(\alpha)=\infty$ for $\alpha \notin\left[d_{-}, d_{+}\right]$.

In the interval $\left(d_{-}, d_{+}\right)$the map $H(\alpha)$ is infinitely differentiable and strictly convex unless $g$ is constant $P$-a.s.; we have $H^{\prime}(\alpha)=\mathbb{E}_{P_{\alpha}}[g]$ and $H^{\prime \prime}(\alpha)=\operatorname{Var}_{P_{\alpha}}(g)$.

(b) The map $L(\delta)$ is a convex, non-negative and lower semi-continuous function of $\delta$ and $L\left(\mathbb{E}_{P}[g]\right)=0$. If $g$ is not constant $P$-a.s. then $L(\delta)$ is strictly convex in the interval $\left(H^{\prime}\left(d_{-}\right), H\left(d_{+}\right)\right)$and for any $\delta \in\left(H^{\prime}\left(d_{-}\right), H\left(d_{+}\right)\right)$there exists $\alpha \in\left(d_{-}, d_{+}\right)$such that $H^{\prime}(\alpha)=\delta$ and

$$
L(\delta)=\alpha H^{\prime}(\alpha)-H(\alpha)=\mathcal{R}\left(P_{\alpha} \| P\right) .
$$

Proof. These are standard results used in the theory of large deviations, see e.g. [29, Lemma 2.2.5, Exercise 2.2.24].

We turn next to the proof of Proposition 3 Most ingredients in the proof have appeared in various recent papers by (some of) the authors and their collaborators [26, 28, 25]. The formulation here is slightly different and since the results play a central role in the paper we provide a proof for convenience and completeness.

Proof of Proposition 3. First note that it is enough to prove the result for $H(\alpha)$ since the result for $H(-\alpha)$ is obtained by replacing $g$ by $-g$.

We first claim that automatically

$$
H\left(d_{+}\right)=\lim _{\alpha \uparrow d_{+}} H(\alpha),
$$

where $H\left(d_{+}\right)$may be infinite. By monotone convergence

$$
\mathbb{E}_{P}\left[1_{\{g \geq 0\}} e^{\alpha g}\right] \uparrow \mathbb{E}_{P}\left[1_{\{g \geq 0\}} e^{d_{+} g}\right]
$$

as $\alpha \uparrow d_{+}$. By dominated convergence

$$
\mathbb{E}_{P}\left[1_{\{g<0\}} e^{\alpha g}\right] \downarrow \mathbb{E}_{P}\left[1_{\{g<0\}} e^{d_{+} g}\right]
$$

as $\alpha \uparrow d_{+}$, and the claim follows. A very similar argument shows that $H^{\prime}(\alpha)$ also has a limit as $\alpha \uparrow d_{+}$.

Let

$$
B(\alpha ; M)=\frac{H(\alpha)+M}{\alpha} .
$$

We divide into cases. Always we have $H(0)=0$. 
1. $g_{+}<\infty$. In this case $H^{\prime}(\alpha) \uparrow g_{+}<\infty$ as $\alpha \rightarrow \infty$ and $H^{\prime}(0)<g_{+}$. If $M=0$ then the infimum is $H^{\prime}(0)$ and attained at $\alpha_{+}=0$. If $M>0$ then let

$$
B^{\prime}(\alpha ; M)=\frac{\alpha H^{\prime}(\alpha)-H(\alpha)-M}{\alpha^{2}}
$$

for $\alpha \geq 0$. The function $\alpha H^{\prime}(\alpha)-H(\alpha)$ strictly increases from 0 at $\alpha=0$ to some limit $M_{+}>0$ at $\alpha=\infty$, and the minimizer is at the unique finite root of $\alpha H^{\prime}(\alpha)-H(\alpha)=M$ for $M<M_{+}$and $\alpha_{+}=\infty$ for $M \geq M_{+}$.

2. $g_{+}=\infty$. In this case there are two subcases.

(a) $d_{+}=\infty$. In this case since $g_{+}=\infty$ we have $H^{\prime}(\alpha) \uparrow \infty$ as $\alpha \rightarrow \infty$ and $\alpha H^{\prime}(\alpha)-H(\alpha) \rightarrow \infty$ as $\alpha \rightarrow \infty$. Since $0 H^{\prime}(0)-H(0)=0$, in all cases of $M \geq 0$ there is a unique root to $\alpha H^{\prime}(\alpha)-H(\alpha)=M$ and hence a unique minimizer.

(b) $d_{+}<\infty$. We know that $H^{\prime}(\alpha)$ converges as $\alpha \uparrow d_{+}$to a well defined left hand limit which we call $H^{\prime}\left(d_{+}\right)$ (note that this value could be $\infty$ ). Thus we have that $\alpha H^{\prime}(\alpha)-H(\alpha)$ ranges from 0 at $\alpha=0$ to $M_{+}=$ $d_{+} H^{\prime}\left(d_{+}\right)-H\left(d_{+}\right)$. For $M \in\left[0, M_{+}\right)$there is a unique minimizer in $\left[0, d_{+}\right)$. For $M \geq M_{+}$the unique minimizer is at $\alpha_{+}=d_{+}$.

To conclude the proof we note that if $\alpha_{+}<d_{+}$then

$$
\alpha_{+} H^{\prime}\left(\alpha_{+}\right)-H\left(\alpha_{+}\right)=\mathcal{R}\left(P_{\alpha_{+}} \| P\right)=M,
$$

and thus

$$
B\left(\alpha_{+}, M\right)=H^{\prime}\left(\alpha_{+}\right)=\mathbb{E}_{P_{\alpha_{+}}[g]}
$$

which proves (9) and (10). Finally if $d_{+}=\infty$ and $g$ is $P$-a.s. bounded above then the infimum is equal to $\lim _{\alpha \rightarrow \infty} \frac{H(\alpha)}{\alpha}$ and this establishes (12). If $d_{+}<\infty$ and $M_{+}<\infty$ then the bound takes the form (11).

\section{References}

[1] H. Pham. Some Applications and Methods of Large Deviations in Finance and Insurance, pages 191-244. Springer Berlin Heidelberg, 2007.

[2] G. Rubino and B. Tuffin, editors. Rare Event Simulation using Monte Carlo Methods. Wiley, New York, 2009.

[3] W. Cai, M.H. Kalos, M. de Koning, and V.V. Bulatov. Importance sampling for rare transition events in Markovian processes. Phys. Rev. E, 66:046703, 2002.

[4] P. Embrechts, C. Klppelberg, and T. Mikosch. Modelling Extremal Events for Insurance and Finance. Springer-Verlag Berlin Heidelberg, 1997.

[5] S. Asmussen and P.W. Glynn. Stochastic simulation: algorithms and analysis. Stochastic modelling and applied probability. Springer, New York, 2007.

[6] R. Y. Rubinstein and D. P. Kroese. Simulation and the Monte Carlo method. Wiley Series in Probability and Statistics. John Wiley \& Sons, Inc., Hoboken, NJ, 2017. Third edition.

[7] P. Dupuis and H. Wang. Subsolutions of an Isaacs equation and efficient schemes for importance sampling. Math. Oper. Res., 32:1-35, 2007.

[8] P. Del Moral. Feynman-Kac formulae. Probability and its Applications (New York). Springer-Verlag, New York, 2004. Genealogical and interacting particle systems with applications.

[9] P. Del Moral and J. Garnier. Genealogical particle analysis of rare events. Ann. Appl. Probab., 15(4):2496-2534, 2005.

[10] P. L'Ecuyer, F. Le Gland, P. Lezaud, and B. Tuffin. Splitting Techniques, pages 39-61. John Wiley \& Sons, Ltd, 2009.

[11] M. Villen-Altamirano and J. Villen-Altamirano. Restart: A method for accelerating rare event simulations. In Proc. of the 13th International Teletraffic Congress, Queueing, Performance and Control in ATM, pages 71-76, Amsterdam, 1991. Elsevier. 
[12] M.J.J. Garvels. The Splitting Method in Rare Event Simulation. PhD thesis, University of Twente, The Netherlands, 2000 .

[13] T. Dean and P. Dupuis. The design and analysis of a generalized RESTART/DPR algorithm for rare event simulation. Annals of OR, 189:63-102, 2011.

[14] J. S. Liu. Monte Carlo strategies in scientific computing. Springer Series in Statistics. Springer-Verlag, New York, 2001.

[15] D. Frenkel and B. Smit. Understanding Molecular Simulation, From Algorithms to Applications. Academic Press, 2002.

[16] O. Valsson, P. Tiwary, and M. Parrinello. Enhancing important fluctuations: Rare events and metadynamics from a conceptual viewpoint. Annual Review of Physical Chemistry, 67(1):159-184, 2016. PMID: 26980304.

[17] S. Asmussen and R. Y. Rubinstein. Sensitivity analysis of insurance risk models via simulation. Management Science, 45(8):1125-1141, 1999.

[18] F. J. Vazquez-Abad and P. LeQuoc. Sensitivity analysis for ruin probabilities: Canonical risk model. The Journal of the Operational Research Society, 52(1):71-81, 2001.

[19] A. Agarwal, S. De Marco, E. Gobet, and G. Liu. Rare event simulation related to financial risks: efficient estimation and sensitivity analysis. working paper or preprint, October 2015.

[20] P. Del Moral, P. Hu, and L. Wu. On the concentration properties of interacting particle processes. Foundations and Trends in Machine Learning, 3(3-4):225-389, 2012.

[21] R. Atar, K. Chowdhary, and P. Dupuis. Robust bounds on risk-sensitive functionals via rényi divergence. SIAM/ASA Journal on Uncertainty Quantification, 3:18-33, 2015.

[22] S. M. Kay. Fundamentals of Statistical Signal Processing: Estimation Theory. Prentice-Hall, Inc., Upper Saddle River, NJ, USA, 1993.

[23] P. Dupuis, M. Katsoulakis, L. Rey-Bellet, and J. Wang. Uncertainty quantification bounds and Rényi divergence. In preparation.

[24] S. Boucheron, G. Lugosi, and P. Massart. Concentration inequalities: A nonasymptotic theory of independence. Oxford University Press, 2016.

[25] K. Gourgoulias, M. A. Katsoulakis, L. Rey-Bellet, and J. Wang. How biased is your model? Concentration Inequalities, Information and Model Bias. ArXiv e-prints, June 2017.

[26] K. Chowdhary and P. Dupuis. Distinguishing and integrating aleatoric and epistemic variation in uncertainty quantification. ESAIM: Mathematical Modelling and Numerical Analysis, 47:635-662, 2013.

[27] J. Li and D. Xiu. Computation of failure probability subject to epistemic uncertainty. SIAM Journal on Scientific Computing, 34(6):A2946-A2964, 2012.

[28] P. Dupuis, M. A. Katsoulakis, Y. Pantazis, and P. Plecháč. Path-Space Information Bounds for Uncertainty Quantification and Sensitivity Analysis of Stochastic Dynamics. SIAM/ASA Journal on Uncertainty Quantification, 4(1):80$111,2016$.

[29] A. Dembo and O. Zeitouni. Large Deviations Techniques and Applications. Springer, 1998.

[30] F. Liese and I. Vajda. Convex statistical distances, volume 95 of Teubner-Texte zur Mathematik [Teubner Texts in Mathematics]. BSB B. G. Teubner Verlagsgesellschaft, Leipzig, 1987. With German, French and Russian summaries.

[31] A. Rényi. On measures of entropy and information. In Proc. of the 4th Berkeley Symposium on Mathematical Statistics and Probability, volume I, pages 547-561, Berkeley, CA, 1961. University of California Press.

[32] I. Vajda. Distances and discrimination rates for stochastic processes. Stochastic Processes and Applications, 35:47-57, 1990.

[33] T. van Erven and P. Harremoës. Rényi divergence and Kullback-Leibler divergence. IEEE Transactions on Information Theory, 60(7):3797-3820, 2014. 
[34] L. Golshani, E. Pasha, and G. Yari. Some properties of Rényi entropy and Rényi entropy rate. Information Sciences, 179:2426-2433, 2009.

[35] P. Dupuis and R.S. Ellis. A Weak Convergence Approach to the Theory of Large Deviations. Wiley Series in Probability and Statistics, 1997.

[36] B. Simon. The statistical mechanics of lattice gases. Vol. I. Princeton Series in Physics. Princeton University Press, Princeton, NJ, 1993.

[37] M.A. Katsoulakis, L. Rey-Bellet, and J. Wang. Scalable information inequalities for uncertainty quantification. Journal of Computational Physics, 336:513-545, 2017.

[38] G. Arampatzis, M. A. Katsoulakis, and Y Pantazis. Accelerated sensitivity analysis in high-dimensional stochastic reaction networks. PLoS ONE, 10(7):1-24, 072015.

[39] T. Lelievre, M. Rousset, and G. Stoltz. Free energy computations: a mathematical perspective. Imperial College Press, 2010.

[40] C. M. Bishop. Pattern Recognition and Machine Learning. Springer-Verlag New York, Inc., Secaucus, NJ, USA, 2006. 